\title{
The "Big Two" and socially induced emotions: Agency and communion jointly influence emotional contagion and emotional mimicry
}

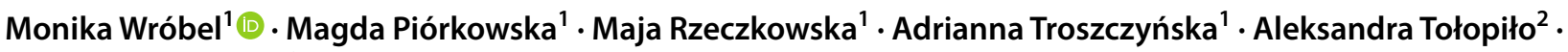 \\ Michał Olszanowski
}

Accepted: 12 June 2021 / Published online: 18 June 2021

(c) The Author(s) 2021

\begin{abstract}
Three studies investigated the effects of two fundamental dimensions of social perception on emotional contagion (i.e., the transfer of emotions between people). Rooting our hypotheses in the Dual Perspective Model of Agency and Communion (Abele and Wojciszke in Adv Exp Soc Psychol 50:198-255, https://doi.org/10.1016/B978-0-12-800284-1.00004-7, 2014), we predicted that agency would strengthen the effects of communion on emotional contagion and emotional mimicry (a process often considered a key mechanism behind emotional contagion). To test this hypothesis, we exposed participants to happy, sad, and angry senders characterized by low vs. high communion and agency. Our results demonstrated that, as expected, the effects of the two dimensions on socially induced emotions were interactive. The strength and direction of these effects, however, were consistent with our predictions only when the senders expressed happiness. When the senders expressed sadness, we found no effects of agency or communion on participants' emotional responses, whereas for anger a mixed pattern emerged. Overall, our results align with the notion that emotional contagion and mimicry are modulated not only by the senders' traits but also by the social meaning of the expressed emotion.
\end{abstract}

Keywords Agency $\cdot$ Communion $\cdot$ Emotional contagion $\cdot$ Emotional mimicry $\cdot$ Emotional expression

Everyday experience and empirical evidence indicate that emotions spread between people. For instance, individuals exposed to emotional displays respond to these displays with congruent emotions, which is often referred to as emotional contagion (Hatfield et al., 1994; Prochazkova \& Kret, 2017). Hatfield et al. (1994), who promoted the term, stated that emotions, similar to diseases, are transferred in a reflex-like manner because the process is triggered by emotional mimicry (i.e., an unconscious, automatic imitation of the sender's emotional display). However, research on the relationship between mimicry and contagion has yielded an inconsistent pattern of findings, with some studies showing that a change in the receiver's inner feelings may indeed be triggered by a change in the receiver's emotional expression

Monika Wróbel

monika.wrobel@uni.lodz.pl

1 Institute of Psychology, University of Lodz, Smugowa 10/12, 91-433 Lodz, Poland

2 Center for Research on Biological Basis of Social Behavior, SWPS University of Social Sciences and Humanities, Warsaw, Poland
(Olszanowski et al., 2020; Sato et al., 2013), while others report no such effect (Hess \& Blairy, 2001; Van der Schalk et al., 2011). This suggests that emotional contagion is more complex than emotional mimicry and hence, can arise from various (more or less automatic) mechanisms (Wróbel \& Imbir, 2019).

Evidence has also accumulated that, despite the label, the spread of emotions is much more selective than the spread of diseases because people avoid sharing the emotions of those whose intents seem non-affiliative (for a review, see Wróbel \& Imbir, 2019). For instance, a friendly or trustworthy sender is perceived as having more affiliative intents than an unfriendly or untrustworthy sender, and thus, the emotional displays of the former are more likely to evoke congruent emotions than the emotional displays of the latter (e.g., Epstude \& Mussweiler, 2009; Wróbel \& Królewiak, 2017). A similar pattern of results has been found for emotional mimicry (for reviews, see Hess \& Fischer, 2013, 2014; Seibt et al., 2015), which suggests that emotional contagion and emotional mimicry, although not always related, are moderated by social factors in a quite comparable way (Wróbel \& Imbir, 2019). Therefore, congruent responses to the sender's 
emotional displays may be manifested by both congruent self-reported emotions (contagion) and congruent facial activity (mimicry).

The notion that emotions are more likely to be shared and imitated when the sender's intents are perceived as affiliative indicates that the receiver's emotional responses to the sender's expressed emotions are controlled by communion, a fundamental dimension of social perception that is intrinsically linked to liking and affiliation (Wojciszke et al., 2009). Yet, a large body of research shows that communion (e.g., being friendly, honest, or warm) represents only one dimension of social perception. The second of the "Big Two", often pushed into the background by communion, is agency, which refers to such traits as being capable, ambitious, or hard-working (Abele \& Wojciszke, 2014). Although empirical evidence shows that both dimensions interact when influencing the receiver's perception of the sender's intents, previous research on emotional contagion and emotional mimicry has neglected this possibility because it was focused on the factors directly linked to liking and affiliation (and thus related to communion). Here, we go a step further by investigating whether the sender's perceived ability to enact his/her intents (i.e., agency) also matters for the magnitude of contagion or mimicry. Three studies reported here tested these joint effects of both social dimensions on the receiver's emotional responses.

\section{Emotional contagion, mimicry, and the interplay between agency and communion}

As already mentioned, emotional contagion and its mechanisms are modulated by the sender's communal traits because such traits indicate the sender's affiliative intents. Yet, when making social judgments, people rely not only on communal but also on agentic information. For instance, Wojciszke et al. (1998) demonstrated that when participants were asked to evaluate other people, communal characteristics contributed to whether the impressions of others were positive or negative, whereas agentic characteristics influenced the intensity of these impressions.

This two-dimensionality of social perception can be explained within the framework of the Dual Perspective Model of Agency and Communion (DPM-AC; Abele \& Wojciszke, 2014). The model holds that the first thing people want to know about the other person is whether this person is high or low in communion because this helps them differentiate between "friends" and "foes". The second thing people want to know is whether this person has competence (e.g., knowledge, abilities, or skills) required to enact these friendly or unfriendly intents, which is manifested by this person's high or low agency, respectively.
In consequence, the influences of agency and communion on social judgments combine in a nonadditive way: When high (vs. low) communion is accompanied by high agency the overall impression of whether the other person acts in affiliative (vs. non-affiliative) way is clear and strong, whereas when high (vs. low) communion is accompanied by low agency this impression becomes weaker (see also Fiske et al., 2007).

Drawing on the Dual Perspective Model of Agency and Communion (Abele \& Wojciszke, 2014) as well as the recent models of emotional contagion and mimicry (Fischer \& Hess, 2017; Wróbel \& Imbir, 2019), we propose that emotional contagion and mimicry are also jointly modulated by both social dimensions. More specifically, high communion, being a signal of the sender's affiliative intents, should foster concordant responses to his/her emotional displays, whereas low communion, being a signal of the sender's nonaffiliative intents, should hinder such responses. This effect, however, should be more pronounced when the sender is high in agency, because high-agency senders, in contrast to low-agency senders, are able to enact their affiliative or non-affiliative intents. Accordingly, the effects of agentic traits on emotional contagion and mimicry should depend on their interaction with communal traits. When communion is high, high agency should foster the receiver's concordant responses to the sender's emotional displays, whereas when communion is low high agency should hinder these responses.

Our reasoning is indirectly supported by studies showing that the role of status and power (i.e., two variables tightly linked to agency; Wojciszke et al., 2009) in the transfer of emotions between people may vary depending on whether contextual information highlights the sender's high or low communion. Specifically, studies that demonstrated that the sender's high status or power fostered the induction of concordant emotions following exposure to the sender's emotional displays were run in the contexts associated with high communion (e.g., among partners or roommates; Anderson et al., 2003). Studies that led to contradictory findings, that is, showed that emotions displayed by low-status or low-power senders are more "contagious" than emotions displayed by high-status or high-power senders, were conducted in the contexts associated with low communion. For instance, participants were assigned to the roles of leaders or followers and led to believe that the followers should obey the leaders because the leaders scored higher on the leadership skills questionnaire (Spoor \& Kelly, 2009) or could punish the followers by administering electric shocks (Hsee et al., 1990). Although none of these studies measured or manipulated the sender's agentic or communal traits directly, the fact that status and power played opposite roles in highvs. low-communal social contexts suggests that the effects 
of the sender's agentic traits on the receiver's responses may vary depending on the sender's communal traits.

We are aware of only one study that explicitly tested this possibility. Wróbel et al. (2020) manipulated the sender's traits and demonstrated that high agency intensified the effects of communion on emotional contagion. Specifically, when high communion was combined with high agency, happiness contagion was fostered, that is, exposure to the happy sender led to increased happiness. Yet, when high communion was combined with low agency, no significant change in the receiver's self-reported happiness following exposure to the happy sender was observed. Furthermore, when low communion was combined with low agency, no significant change in the receiver's self-reported happiness occurred, whereas when low communion was combined with high agency, happiness levels in response to the happy sender decreased, leading to countercontagion (Hatfield et al., 1994). Interestingly, though, no similar effects were found when the sender was sad (i.e., participants "caught" sadness regardless of the sender's traits), which-as noted by the authors - might have been related to the pre- and poststudy design. This design might have focused participants' attention on how much their emotions had changed following exposure to the sender's emotional display. This, in turn, might have contributed to biased results in the case of sadness, because, in an attempt to conform to social demands, participants did not want to appear insensitive to a sad person regardless of this person's traits. Nevertheless, the question remains whether the effects of agency and communion on the receiver's responses vary across expressed emotions. This is a key question here because different emotional expressions communicate different social messages (Fischer \& Manstead, 2008; Hareli \& Hess, 2012) and thus, may be more or less likely to evoke congruent emotional responses.

\section{Emotional contagion and the social meaning of the expressed emotion}

The senders' affiliative vs. non-affiliative intents may be signaled not only by their traits, but also by the emotion they express. This idea, rooted in the social functional approach to emotions, holds that emotions are social signals and thus may either strengthen relationships with others or keep others at a distance (Fischer \& Manstead, 2008; Keltner \& Haidt, 1999). The affiliation function is played by emotions that help an individual to establish or maintain harmonious relations with others. Thus, it is typical of positive emotions that signal the intention to build, restore, or maintain social bonds (e.g., happiness, gratitude), but some negative emotions (e.g., sadness, guilt) also serve social bonding by informing others that one wants to establish or repair a social relationship. For instance, sadness signals helplessness and need for support and thus, may evoke the intention to offer help or consolation (Van Kleef et al., 2011). The social distancing function serves to differentiate or distance oneself from others or compete with these others for social status. This function is typical of negative emotions that signal the intention to attack, exclude, move away from, or isolate others (e.g., anger, fear, contempt), but some positive emotions (e.g., pride about oneself) may also weaken social bonds (Fischer \& Manstead, 2008).

The social-signaling function of the sender's expressed emotion may further affect the tendency to share and mimic this emotion (Fischer \& Hess, 2017; Hess, 2021; Wróbel $\&$ Imbir, 2019). For instance, when no contextual information is given, smiling senders are, by default, perceived as having more affiliative intents than frowning senders and thus smiles are more likely to evoke congruent emotional reactions than frowns (for a review, see Fischer \& Hess, 2017). Put differently, when provided with no additional information about the sender, the knowledge about his/her intents may be inferred from the intrinsic meaning of the expressed emotion. Yet, when such information is given, it may affect the meaning of the sender's expression and modulate its potential to evoke congruent emotional reactions (Hess, 2021; Hess \& Fischer, 2014). For instance, although a smile in itself is an affiliative social signal that is readily shared and mimicked, its meaning changes to non-affiliative and its "contagiousness" decreases when it is shown by a low-communion sender (e.g., a rival or dislikable person; Likowski et al., 2008; Weyers et al., 2009; Wróbel \& Królewiak, 2017). Contextual information may also affect the meaning of intrinsically non-affiliative emotions, which in turn, may strengthen their potential to evoke congruent responses. For instance, although in general people avoid imitating and sharing anger, in some contexts (e.g., when the receiver knows that the sender's anger is directed at a person the receiver does not like), the display of anger may be more readily mimicked (Bourgeois \& Hess, 2008). At the same time, previous findings on the role of the senders' characteristics in modulating the receivers' responses to the expressions of anger are highly inconclusive, with some studies showing that angry displays are more likely to elicit anger in affiliative social context (e.g., when the sender is an ingroup member; Weisbuch \& Ambady, 2008), while others report the opposite effect (e.g., show that outgroup anger is mimicked to a greater extent; van der Schalk et al., 2011).

Overall, much evidence shows that emotional contagion and mimicry are complex social phenomena shaped by the interplay of the sender's characteristics and the meaning of the expressed emotion. Thus, as shown in Fig. 1, we think that the effects of agency and communion on the receiver's responses to the sender's emotional displays may also vary across intrinsically affiliative vs. non-affiliative emotions. More specifically, we expect that the sender's agentic and 
Fig. 1 The hypothetical effects of the sender's expressed emotion, communal and agentic traits on the receiver's tendency to share and mimic the sender's emotion

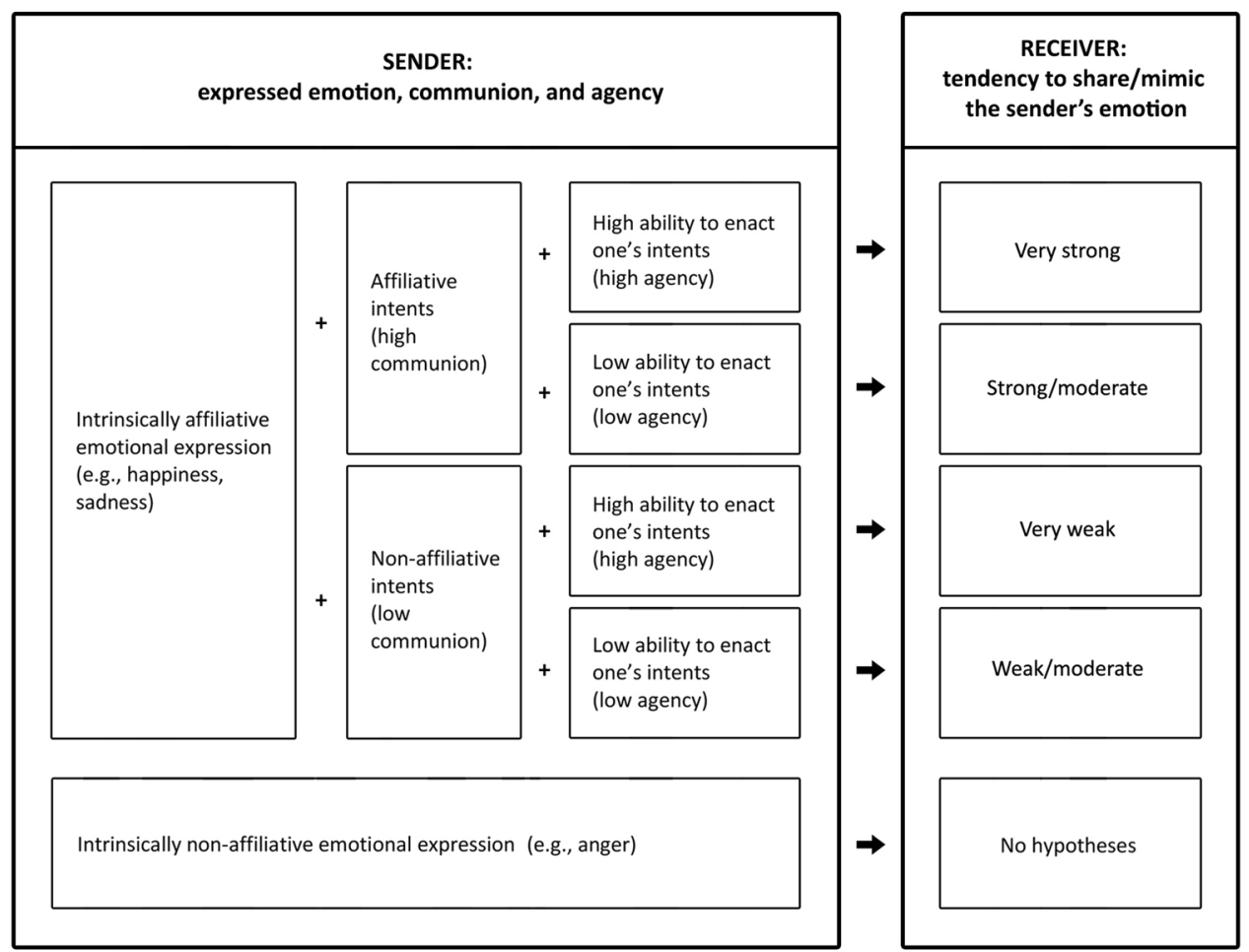

communal traits will modulate the receiver's tendency to react congruently to the sender's intrinsically affiliative displays (e.g., happiness and sadness). Such displays, when no additional information about the sender is provided, typically evoke congruent responses and thus the sender's traits will reinforce or reduce this tendency: High communion, by strengthening the affiliation signals sent by the sender's expression, will boost its potential to evoke congruent responses, while low communion by contradicting these signals, will weaken its potential to evoke congruent responses. We also expect that the tendency to share and imitate the sender's intrinsically affiliative emotions will be further reinforced (vs. weakened) by high (vs. low agency), which will make the effects of communion more (vs. less) pronounced.

We do not formulate any directional hypotheses concerning non-affiliative expressions (e.g., anger) because such expressions per se signal antagonism and thus, findings on the imitation of these expressions are mixed, with many studies showing limited evidence for mimicry or contagion (for reviews, see Hess \& Fischer, 2013; Wróbel \& Imbir, 2019). Moreover, the displays of intrinsically non-affiliative emotions frequently elicit so called reactive responses rather than mimicry/contagion responses (Elfenbein, 2014; Hess $\&$ Fischer, 2013). For instance, when anger is perceived as threatening (which in the case of anger happens very often), it evokes reactive fear and anger (Rees et al., 2020; Springer et al., 2007). Such reactive responses (complementary and convergent, respectively) are different from mimicry and contagion, because the receiver reacts to the perceived threat rather than shares the same anger (Elfenbein, 2014). Accordingly, when reactive responses to anger occur, the possibility of imitating and sharing the senders' expressed emotions decreases, because mimicry or contagion tend to occur when the sender's intents are affiliative (Hess \& Fischer, 2013). At the same time, convergent reactive responses may be easily confused with mimicry/contagion because they look similar (Wróbel \& Imbir, 2019), which adds to the complexity of the picture and makes the role of agency and communion in the social induction of anger even more difficult to predict.

\section{Overview of studies}

Extending prior work on moderators of emotional contagion and mimicry, the present research examined the joint effects of the two fundamental dimensions of social perception on socially induced emotional responses. Thus far, such effects have been tested in only one study that focused on affiliative emotional displays, that is, happiness and sadness (Wróbel et al., 2020). Here, we used both affiliative and non-affiliative emotional displays to test whether the effects of agency and communion on the receiver's responses vary depending on the intrinsic affiliative meaning of the expressed emotion. To that aim, we chose happiness and anger, because these two emotional displays differ markedly in their social meaning and are most often studied in the context of emotional contagion and mimicry (for reviews, see Hess \& Fischer, 2013; Seibt et al., 2015). Moreover, as we were interested in 
emotion-specific effects rather than valence-specific effects, we decided to include one more emotion that is similar to anger in terms of valence, and similar to happiness in terms of affiliative social meaning. In consequence, we added sadness because it meets both criteria and is often included in studies on emotional contagion and mimicry (e.g., Hess \& Blairy, 2001; Likowski et al., 2008).

We also addressed two other limitations of the study by Wróbel et al. (2020). First, the authors used videos showing one videotaped man, which reduces the possibility to generalize their findings to female senders. In the present research, we used videos showing both men and women. Second, Wróbel et al. (2020) relied exclusively on self-report measures of emotion that provide no information about emotional mimicry and may be biased by social demands (Robinson $\&$ Clore, 2002). Thus, as already mentioned, to conform to these demands, participants exposed to the sad sender (i.e., a person whose emotional display signaled helplessness and need to support) might have been reluctant to admit that their emotions did not change, regardless of this person's traits. This may explain the null effects of the sender's traits on sadness contagion. Therefore, in Studies 2 and 3, we went beyond self-reported emotions (i.e., contagion) and tested the additional effects of agency and communion on facial muscle activity (i.e., mimicry). Moreover, to reduce participants' focus on the increase or decrease in each of their selfreported emotions, we exposed participants to twelve (Studies 1 and 3) or eight (Study 2) videos presented in random order and measured how participants felt after each of them, instead of using just one video and a pre- and post-measure.

The research received approval from the Ethics Committee of the University of Lodz, Poland. All participants provided written informed consent and were entered into a raffle for gift cards (the equivalent of approx. $\$ 10, \$ 12$, and \$15.00; Studies 1 and 2) or participated in exchange for partial course credit and coffee vouchers (the equivalent of approx. \$5; Study 3). Our design and analysis plan were preregistered prior to the start of data collection and deviations from the pre-registered protocol are explicitly mentioned. All measures, manipulations, and exclusions are fully disclosed. Data, stimuli and preregistrations are available at https://osf.io/akmdc/.

\section{Study 1}

To provide initial evidence for the joint effects of agency and communion on socially induced emotions, we manipulated the senders' agentic and communal traits and then exposed participants to the senders' displays of happiness, sadness, and anger. We expected that happy and sad expressions would evoke congruent emotional responses (that is, participants' emotions would correspond to emotions expressed by the senders). We also hypothesized that these congruent responses would be promoted by high communion and weakened by low communion. Finally, we expected that high agency would intensify the effects of communion on participants' responses to happiness and sadness, whereas low agency would lessen these effects. As previously mentioned, we did not formulate any specific hypotheses concerning anger-related responses.

\section{Method}

\section{Participants and design}

We used G*Power (Faul et al., 2007) and the results of the previous study on the effect of agency and communion on emotional contagion (Wróbel et al., 2020), to calculate the sample size. ${ }^{1}$ The analysis indicated that $n=58$ was required to detect an effect size of $f=.20$ with $95 \%$ power and alpha level of .05 . We recruited 72 undergraduates ( 52 women) but based on predetermined exclusion criteria we excluded participants who guessed the hypotheses $(n=2)$ or declared that they had not watched the videos carefully $(n=8)$. Our final sample included 62 participants (41 women; $M_{\text {age }}=21.74$, years, $S D=4.21$ ). We used a 3 (senders' emotional display: happiness, sadness, anger) $\times 2$ (senders' communal traits: high communion, low communion) $\times 2$ (senders' agentic traits: high agency, low agency) within-participants design.

\section{Procedure and materials}

Participants were scheduled for individual sessions. To divert their attention from the real aim of the research, they were informed that the study would be about the relationship between memory and first impressions. After signing the informed consent, each participant was seated at a computer and asked to follow the instructions on the screen.

First, participants saw profiles of four senders (two men and two women) shown in random order. Each profile was presented on a separate page and consisted of a picture of a sender showing a neutral facial expression and eight adjectives (each of them in a separate line). Four adjectives referred to the sender's communal traits, while the other four referred to his/her agentic traits. The images were taken from the Warsaw Set of Emotional Facial Expression Pictures (Olszanowski et al., 2015), whereas the traits were taken

\footnotetext{
1 When registering our studies, we were not aware that $G^{*}$ Power cannot be conveniently used for repeated measures designs with more than one within-subject factor. Therefore, we additionally verified our calculations in a post-hoc manner, using PANGEA (Westfall, 2016). The analysis confirmed that the final samples in all three studies were sufficient to detect a small-sized three-way interaction with power $>80 \%$.
} 
from Wojciszke et al. (2009, Study 3). Depending on the experimental condition, the traits represented high vs. low communion (envious, malicious, stingy, unfair vs. helpful, kind, non-egoistic, selfless) and high vs. low agency (competent, educated, hard-working, well-organized vs. disorganized, inert, passive, undependable), which resulted in four possible combinations of traits. The allocation of the four combinations to the four senders as well as the order of the traits in each combination were random. To make the profiles more credible, participants were led to believe that the four individuals presented in the pictures had been anonymously assessed by other people and that the traits below each picture had been most often mentioned in these assessments. Participants were also asked to memorize the profiles for later recall tasks and thus, the presentation time was not limited (i.e., participants moved to the next profile once they felt ready).

To ensure that participants associated the profiles with the right senders, we administered the first recall task. The images of the senders were shown once again and participants were asked whether each person was described by communal (friendly, nice, $r_{S B}=.91$ ) and agentic (efficient, competent, $r_{S B}=.74$ ) characteristics. Ratings were made on a 7-point scale ranging from definitely no to definitely yes.

Next, participants were exposed to twelve 35-s videos presenting the senders expressing happiness, sadness, and anger ( 4 senders $\times 3$ displayed emotions). ${ }^{2}$ The videos were presented in random order and showed dynamic facial displays created by morphing the images we used at the beginning of the study (i.e., neutral faces of the senders) with the images of anger, sadness, and happiness displayed by the same four senders (Olszanowski et al., 2020). The frame rate in each video was 29 frames per second. Each video started with a 2-s still image of a neutral face that within $3 \mathrm{~s}$ changed gradually to a full emotional display (apex). After reaching the apex, the face returned within the next $3 \mathrm{~s}$ to a blended expression (half-neutral, half-emotional), and then, after $3 \mathrm{~s}$, it reached the apex again. The transformations were repeated four times. The video finished with a still image of a blended expression.

After each video participants rated the extent to which they felt happiness (happy, cheerful, delighted; $\omega=.92$ ), sadness (sad, downhearted, blue; $\omega=.87$ ), anger (angry, irritated, mad, $\omega=.82$ ) and fear (anxious, fearful, tense, $\omega=.81$ ) on a scale ranging from 1 (not at all) to 7 (extremely). The items were taken from a modified Polish version of the

\footnotetext{
2 The length of the videos was based on the observation that videos used in a typical emotional contagion study are much longer (from 1 to $3 \mathrm{~min}$ ) than videos used in a typical emotional mimicry study (from 1 to $5 \mathrm{~s}$; see Wróbel \& Olszanowski, 2019). Previous research confirmed that the videos successfully induce congruent emotional responses (Olszanowski et al., 2020; Wróbel \& Olszanowski, 2019).
}

Differential Emotion Scale (DES; Izard et al., 1974) and presented in random order. We decided to measure fear in addition to the emotions that directly corresponded to the senders' emotional displays, because as already mentioned, expressed anger often elicits reactive anger and fear rather than mimicry/contagion (see also Dimberg \& Thunberg, 2012; Wróbel \& Olszanowski, 2019). The identification of reactive responses to anger is important because, their occurrence suggests that the angry senders' intents are perceived as non-affiliative and thus, the possibility of sharing the senders' expressed emotions (via mimicry and/or contagion) decreases (Hess \& Fischer, 2013; Wróbel \& Imbir, 2019). However, as we did not have any predictions concerning participants' responses to anger, we did not have any hypotheses not only for self-reported anger, but also for self-reported fear.

Having watched the videos, participants performed the second recall task $\left(r_{S B \text { communion }}=.75 ; r_{S B \text { agency }}=.74\right)$. Lastly, they were asked about their assumptions regarding the goals of the study and whether they had watched the videos carefully.

\section{Results and discussion}

\section{Manipulation check}

Planned contrasts confirmed that the trait manipulation was successful. The high-communion senders were rated as more communal $(M=5.86, S D=0.96)$ than the low-communion senders $(M=1.94, S D=0.84), F(1,61)=394.82, p<.001$, $\eta_{\mathrm{p}}{ }^{2}=.87[.80, .90]$ and the high-agency senders as much more agentic $(M=6.05, S D=0.99)$ than the low-agency senders $(M=2.16, S D=1.02), F(1,61)=312.90, p<.001$, $\eta_{\mathrm{p}}^{2}=.84[.76, .88]$. Means and standard deviations for all conditions are given in the Supplementary Table S2.

\section{Emotional contagion}

Next, we assessed the influence of the senders' emotional displays and traits on happiness contagion, using a 3 (senders' emotional display: happiness, anger, sadness) $\times 2$ (senders' communal traits: high communion, low communion $) \times 2$ (senders' agentic traits: high agency, low agency) repeated measures ANOVA, with self-reported happiness as a dependent variable. The analysis showed the expected threeway interaction, $F(2,122)=4.44 ; p=.014 ; \eta_{\mathrm{p}}{ }^{2}=.07[.003$, .16] (see Fig. 2, Panel A). We then run a series of planned contrasts consistent with our hypotheses. Contrast weights are given in Table 1. The first contrast compared happiness levels following exposure to the happy senders $(M=4.86$, $S D=1.26$ ) with happiness levels following exposure to the sad and angry senders $(M=1.87, S D=1.01)$. The contrast was significant, $F(1,61)=187.09 ; p<.001 ; \eta_{\mathrm{p}}{ }^{2}=.75[.64$; 
Panel A

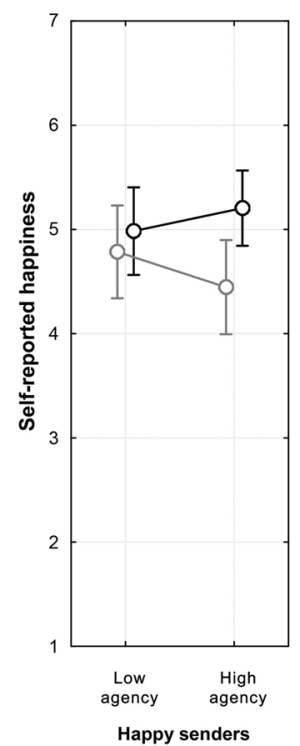

Panel C

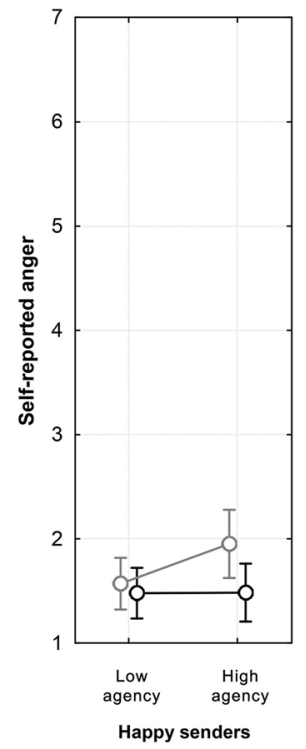

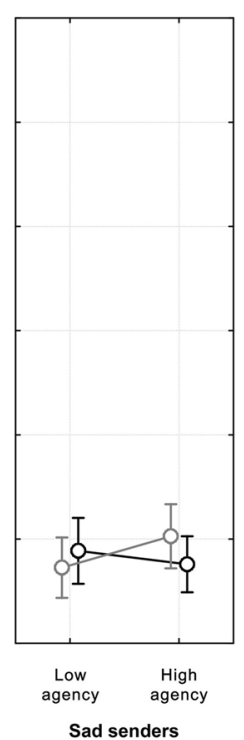

Sad senders

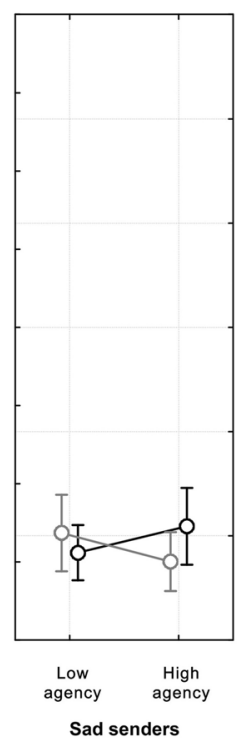

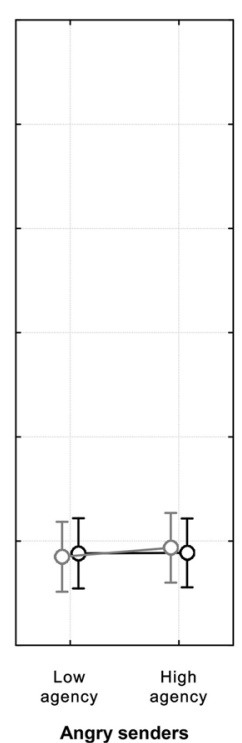

Angry senders

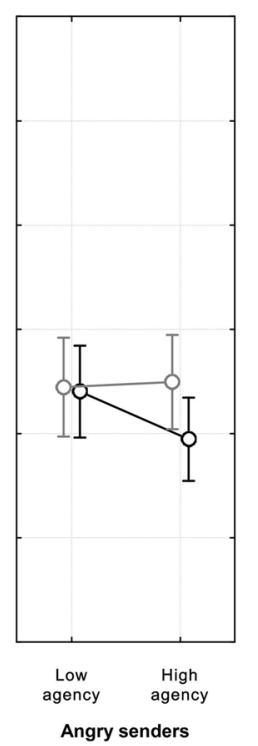

$-0-$ Low

communion

Fig. 2 Participants' self-reported happiness (A), sadness (B), anger $(\mathbf{C})$, and fear (D) as a function of the senders' communal traits, agentic traits and emotional expression (Study 1). Scores range from 1 to

.82], indicating that we observed the induction of convergent emotion (happiness contagion). The second contrast tested whether happiness contagion was moderated by the senders' communion. This contrast was also significant, $F(1,61)=8.43 ; p=.005 ; \eta_{\mathrm{p}}{ }^{2}=.12[.01 ; .28]$, indicating that participants reported being significantly more happy after seeing the happy senders high in communion $(M=5.09$,
Panel B
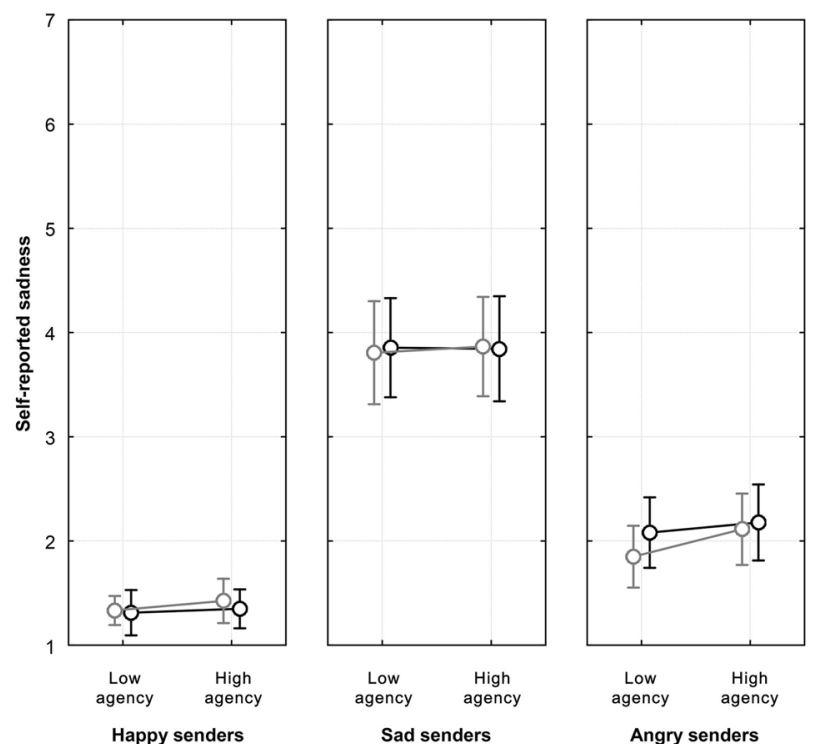

Sad senders

Angry senders

\section{Panel D}
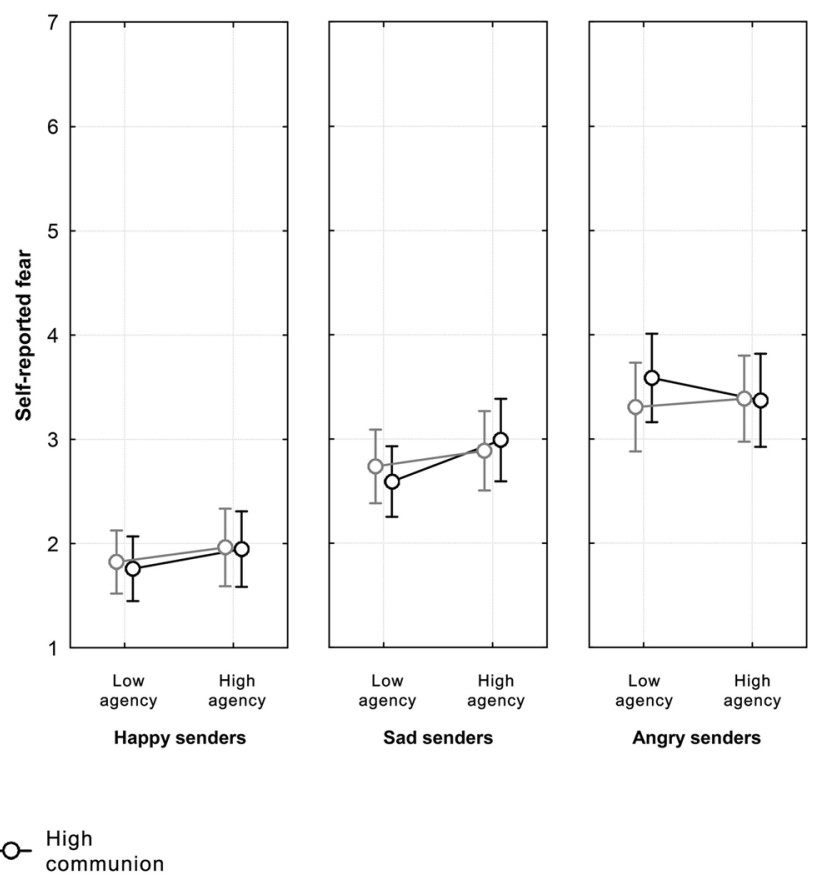

7, with higher scores indicating higher levels of self-reported emotions. Error bars represent $95 \%$ confidence intervals

$S D=1.28)$ than after seeing the happy senders low in communion $(M=4.62, S D=1.54)$. The effect of communion on happiness contagion was further modulated by the senders' agency, as indicated by the last two contrasts. Specifically, for the senders high in agency, communion affected happiness contagion, such that high communion was associated with more happiness $(M=5.20, S D=1.42)$ than low 
Table 1 Contrast weights for the effects of the senders' emotional display, agency, and communion on self-reported happiness (Study 1)

\begin{tabular}{|c|c|c|c|c|c|c|c|c|c|c|c|c|}
\hline \multirow[t]{3}{*}{ Contrasts } & \multicolumn{4}{|c|}{ Happiness display } & \multicolumn{4}{|c|}{ Sadness display } & \multicolumn{4}{|c|}{ Anger display } \\
\hline & \multicolumn{2}{|l|}{ Low $\mathrm{C}$} & \multicolumn{2}{|l|}{ High C } & \multicolumn{2}{|l|}{ Low $\mathrm{C}$} & \multicolumn{2}{|l|}{ High C } & \multicolumn{2}{|l|}{ Low $\mathrm{C}$} & \multicolumn{2}{|l|}{ High C } \\
\hline & Low A & High A & Low A & High A & Low A & High A & Low A & High A & Low A & High A & Low A & High A \\
\hline 1 & +2 & +2 & +2 & +2 & -1 & -1 & -1 & -1 & -1 & -1 & -1 & -1 \\
\hline 2 & +1 & +1 & -1 & -1 & 0 & 0 & 0 & 0 & 0 & 0 & 0 & 0 \\
\hline 3 & 0 & +1 & 0 & -1 & 0 & 0 & 0 & 0 & 0 & 0 & 0 & 0 \\
\hline 4 & +1 & 0 & -1 & 0 & 0 & 0 & 0 & 0 & 0 & 0 & 0 & 0 \\
\hline
\end{tabular}

Low $C$ low communion, High C high communion, Low A low agency, High A high agency

communion $(M=4.45, S D=1.78), F(1,61)=13.38, p<.001$, $\eta_{\mathrm{p}}^{2}=.18[.04, .34]$; for the senders low in agency, communion did not influence happiness contagion (high communion: $M=4.98, S D=1.65$ vs. low communion: $M=4.78$, $S D=1.75), F(1,61)=0.62, p=.434, \eta_{\mathrm{p}}^{2}=.01[0, .11]$.

Taken together, these findings are consistent with the Dual Perspective Model of Agency and Communion, by showing that the effect of communion on happiness contagion was cushioned by the senders' low agency. Possibly the combination of happiness, high communion and low agency resulted in ambivalent social judgements, that is, the senders were seen as having affiliative intents (due to happy expression and high communion) but also as lacking competence required to act according to these intents (due to low agency). This, in turn, might have eliminated the effect of communion on happiness contagion for the low-agency senders.

An analogous analysis for self-reported sadness did not support the predicted three-way interaction, $F(2,122)=.07$; $p=.937 ; \eta_{\mathrm{p}}{ }^{2}=.001[0, .02]$ (Fig. 2, Panel B). Only the first contrast was significant indicating that participants reported higher levels of sadness after seeing the sad senders $(M=3.84, S D=1.49)$ than after seeing the happy and angry senders $(M=1.71, S D=0.79), F(1,61)=128.26$; $p<.001 ; \eta_{\mathrm{p}}{ }^{2}=.68[.53, .76]$. The remaining contrasts testing the role of agency and communion on sadness contagion were not significant $\left(F_{\mathrm{s}}<0.05, p \mathrm{~s}>.827\right)$. Put differently, we observed sadness contagion, but contrary to our hypotheses, participants shared the senders' sadness regardless of agentic and communal traits.

Importantly, the pattern suggesting that agency and communion moderated happiness contagion but did not influence sadness contagion replicates the study by Wróbel et al. (2020). One reason might be that affiliative signals sent by sadness override the information provided by the traits. Sadness, as mentioned above, communicates the need for support (Fischer \& Manstead, 2008) and thus, arouses compassion and triggers the intention to offer social support (Van Kleef et al., 2011). Thus, it is likely that this unique social meaning of sadness counteracts the effects of the senders' non-affiliative traits on sadness contagion. This reasoning is consistent with other studies showing that sad faces often elicit congruent emotions regardless of the sender's characteristics (e.g., Wróbel \& Królewiak, 2017). Still, it is important to note that these studies, similar to the current study, often rely on self-reports. Thus, even though we tried to reduce the impact of social demands by using a different design than Wróbel et al. (2020), it is possible that participants might have been reluctant to admit that they were insensitive to other people's sadness. This interpretation is indirectly supported by studies on empathy which show that self-report measures of empathic responses often do not indicate how people actually feel but rather reflect their knowledge of how they are expected to feel (Zhou et al., 2003). Therefore, the null effects of agency and communion on sadness contagion should be treated with caution.

We then analyzed participants' responses to anger focusing on self-reported anger and fear, that is, two emotions typically evoked by angry expressions. As this analysis was driven not by specific directional predictions but by research questions that corresponded to the hypotheses we formulated for self-reported happiness and sadness, we did not analyze all possible effects in a completely exploratory manner but focused on the magnitude of the effects corresponding to these questions.

For self-reported anger, the analysis revealed a threeway interaction with a medium effect size $\left(\eta_{\mathrm{p}}{ }^{2}=.06[.001\right.$, .15]) (Fig. 2, Panel C). Participants reported being more angry following exposure to anger displays $(M=3.32$, $S D=1.41)$ than following exposure to happiness and sadness displays $(M=1.77, S D=0.75)$, as evidenced by a large effect size of this comparison $\left(\eta_{\mathrm{p}}{ }^{2}=.58[.41, .68]\right)$. We also observed a small-to-medium effect showing that participants responded with less anger to the high-communion angry senders $(M=3.17, S D=1.49)$ than to the low-communion angry senders $(M=3.47, S D=1.61), \eta_{\mathrm{p}}{ }^{2}=.05[0, .19]$. This effect, however, was more pronounced for the high-agency angry senders (high communion: $M=2.95, S D=1.57$ vs. low communion: $M=3.49, S D=1.78 ; \eta_{\mathrm{p}}{ }^{2}=.09$ [.003, .24]), whereas for the low-agency angry senders, the effect 
of communion was small to non-existent (high communion: $M=3.40, S D=1.73$ vs. low communion: $M=3.45$, $\left.S D=1.87 ; \eta_{\mathrm{p}}{ }^{2}<.001[0, .06]\right)$. Overall, these results show that high agency strengthened the effect of communion on participants' self-reported anger following exposure to anger displays, but this effect was opposite to that found for self-reported happiness following exposure to happy displays (that is, the low-communion angry senders evoked more anger than the high-communion angry senders). On the one hand, this result may seem surprising, given that the combination of high communion and high agency suggests that the senders have affiliative characteristics and thus their anger should be less antagonistic and more "contagious" than anger expressed by the senders characterized by other combinations of traits. On the other hand, as previously mentioned, non-affiliative expressions often evoke reactive rather than imitative responses (Fischer \& Hess, 2017). Although such responses are difficult to differentiate from contagion, it seems that in the current study, anger probably elicited such responses, because, when agency was high, participants reported more anger following exposure to the low-communion than to the high-communion angry senders. In the case of contagion, the pattern would have been reversed (i.e., participants would have reported more anger following exposure to the high-communion than to the low-communion angry senders), because contagion is more likely to occur when the sender's intents are perceived as affiliative (Wróbel \& Imbir, 2019).

The analyses for self-reported fear align with the idea that anger expressions evoked reactive responses (Fig. 2, Panel D). Specifically, participants reported higher levels of fear after exposure to anger displays $(M=3.41, S D=1.41)$ than after exposure to happiness and sadness displays $(M=2.34$, $S D=0.89)$, as indicated by the large effect size of this comparison $\left(\eta_{\mathrm{p}}{ }^{2}=.50[.31, .62]\right)$. All other effects, including the three-way interaction were small $\left(\eta_{\mathrm{p}}{ }^{2}<.05\right)$, which suggests that reactive fear in response to anger occurred regardless of the senders' traits.

Summing up, Study 1 provided initial support for the notion that emotional contagion is controlled by agency and communion, but the pattern of results differed substantially across emotions. Specifically, this pattern supported our hypotheses when the senders expressed happiness, but we found no impact of agency or communion on participants' emotional responses when the senders expressed sadness. Moreover, when the senders expressed anger, we probably observed reactive rather than imitative responses.

Two limitations of this study should be noted. First, as already mentioned, due to the use of self-reports, some participants might have been reluctant to admit that they were insensitive to other people's sadness. Second, during the post-session debriefing, some participants mentioned that they found it difficult to focus on the videos, because the session was quite long. Although we excluded those who declared that they had not watched the videos carefully, we cannot be sure whether the remaining participants were fully engaged throughout the study. This seems important because the influence of social factors on emotional contagion may be based on top-down reflective processes (Wróbel \& Imbir, 2019). Accordingly, the lengthy nature of the study might have made participants' less attentive to the senders' characteristics and, in consequence, reduced the impact of agency or communion on participants' responses (see also Sachisthal et al., 2016). We addressed these limitations in Study 2 .

\section{Study 2}

Study 2 was designed to replicate the results of Study 1 . This time we focused on the two emotional displays for which we observed the effects of agency and communion in Study 1 (i.e., happiness and anger). This enabled us to shorten the time needed to complete the experiment. Additionally, we coded participants' facial muscle activity to collect data on not only self-reported emotions (i.e., emotional contagion) but also emotional mimicry.

\section{Method}

\section{Participants and design}

An a priori power analysis, using $\mathrm{G}^{*}$ Power 3.1.3 (Faul et al., 2007 ) indicated that a sample size of 54 was required to detect an effect size of $f=.22$ with $95 \%$ power and alpha level of .05. The effect size was estimated based on the study by Wróbel et al. (2020), because we had not come across a similar study regarding emotional mimicry. Sixty-five undergraduates (43 women) participated in the study. Three participants who indicated that they had not watched the videos carefully $(n=1)$ or guessed the hypotheses $(n=2)$ were excluded. The final sample included 62 participants (41 women; $M_{\text {age }}=20.26$ years, $S D=1.65$ ) but three participants in this sample did not give their consent for the recordings to be used. Therefore, the analyses of facial muscle activity were based on 59 participants (39 women). We used a 2 (senders' emotional display: happiness, anger) $\times 2$ (senders' communal traits: high communion, low communion) $\times 2$ (senders' agentic traits: high agency, low agency) withinparticipants design.

\section{Procedure and materials}

The procedure and materials were identical to those of Study 1 except that we used eight videos instead of 12 (4 senders $\times 2$ displayed emotions) and recorded participants' faces 
using a laptop build-in camera without participants being aware of it. The camera light was covered with the university property sticker so that participants would not realize that the camera was on. ${ }^{3}$ During debriefing, participants were asked whether they were aware of being recorded (no one realized that). They were also asked for consent for the filmed material to be used for further analysis.

In order to make it possible to extract the parts of the recordings in which participants were exposed to the morphed videos, we simultaneously recorded the screen, which enabled us to mark the exact moments when each video started and ended. Next, these extracted parts were manually coded with the Facial Action Coding System (FACS; Ekman et al., 2002). Following other studies (Sachisthal et al., 2016; van der Schalk et al., 2011), we used AU12 (lip corner puller) and AU6 (cheek raiser) for happiness, AU15 (lip corner depressor) for sadness, AU4 (brow lowerer) for anger, and AU5 (upper lid raiser) for fear. We divided the material into three subsets and each subset was coded by an independent FACS-certified coder. ${ }^{4}$ The coders first scanned the recordings for the occurrence of each of the five AUs. If a given $\mathrm{AU}$ did not occur, the score for this $\mathrm{AU}$ was 0 . If the AU was present, the coders rated its intensity, using a following scale: $1=$ low (trace); $2=$ medium (slight or marked); $3=$ high (extreme or maximum). For that aim, they segmented the event into the onset, apex, and offset, and scored the intensity of the apex. If the a given AU occurred more than once during a given recording (i.e., there was more than one onset-apex-offset sequence), only the most intensive apex was coded. When a given AU occurred in combination with other AUs that affected its appearance, the intensity scores were adjusted accordingly. To assess inter-observer reliability, $20 \%$ of recordings were annotated by all three coders. The two-way absolute agreement average-measures ICC initiated good-to-excellent agreement between the coders $(\mathrm{ICC}=.81)$. The two-way absolute agreement single-measures ICC was lower $(\mathrm{ICC}=.59)$ but still indicated fair-to-good agreement between the coders (Cicchetti, 1994; Koo \& Li, 2016).

\footnotetext{
${ }^{3}$ In Poland, property stickers are commonly attached to universityowned items. Moreover, two preliminary studies $(n=12$ and $n=15)$ showed that this method effectively made participants unaware of being recorded.

${ }^{4}$ In the preregistration, we stated that the recordings would be coded by two coders but, as the task was extremally time-consuming, the material was coded by three coders. All of them were trained in rating the intensity of each AU in combination with other AUs based on the FACS manual instructions. As the coders co-authored the study, they were not unaware of the hypotheses. Nevertheless, the risk of biased scores was eliminated because the material was presented in random order and thus, the coders were unaware which conditions they were coding (i.e., what video and what sender a given participant was exposed to).
}

Self-reported happiness $(\omega=.89)$, sadness $(\omega=.87)$, anger $(\omega=.81)$, and fear $(\omega=.86)$ were measured with a modified Polish version of the DES (Izard et al., 1974). Even though participants were not exposed to sadness displays and we had no hypotheses regarding self-reported sadness, we decided to use the same emotion scale as in Study 1 to avoid additional changes in the procedure, thereby making the results easier to compare across the studies. Additionally, participants completed two recall tasks identical to those used in Study 1, rating the senders' communion $\left(r_{S B \text { first task }}=.86 ; r_{S B \text { second task }}=.60\right)$ and agency $\left(r_{S B \text { first task }}=.69 ; r_{S B \text { second task }}=.66\right)$.

\section{Results and discussion}

\section{Manipulation check}

Trait manipulation was successful. Planned contrast showed that participants rated the high-communion senders as more communal $(M=5.74, S D=0.85)$ than the low-communion senders $(M=1.93, S D=0.85), F(1,61)=460.89, p<.001$, $\eta_{\mathrm{p}}{ }^{2}=.88[.82, .91]$, and the high-agency senders as more agentic $(M=6.16, S D=0.75)$ than the low-agency senders $(M=2.14, S D=0.98), F(1,61)=455.81, p<.001, \eta_{\mathrm{p}}{ }^{2}=.88$ $[.82, .91]$. All means and standard deviations are given in Supplementary Table S4.

\section{Emotional contagion}

Similar to Study 1, we first conducted a 2 (senders' emotional display: happiness, anger) $\times 2$ (senders' communal traits: high communion, low communion) $\times 2$ (senders' agentic traits: high agency, low agency) repeated measures ANOVA, with self-reported happiness as a dependent variable. The analysis was followed by a series of planned contrasts. The predicted three-way interaction was significant, $F(1,61)=5.70 ; p=.020 ; \eta_{\mathrm{p}}{ }^{2}=.09[.002, .23]$ (see Fig. 3, Panel A). The first contrast was also significant, indicating that participants reported being more happy following exposure to the happy senders $(M=4.68, S D=1.14)$ than following exposure to the angry senders $(M=2.34, S D=1.24)$, $F(1,61)=145.18 ; p<.001 ; \eta_{\mathrm{p}}{ }^{2}=.70[.57, .78]$. The second contrast confirmed that happiness levels were higher, when the happy senders were high in communion $(M=5.02$, $S D=1.28)$ than low in communion $(M=4.34, S D=1.54)$, $F(1,61)=9.92 ; p=.003 ; \eta_{\mathrm{p}}{ }^{2}=.14[.02, .30]$. Finally, the last two contrasts showed that for the senders high in agency, communion influenced happiness contagion, such that high communion was associated with more happiness $(M=5.07$, $S D=1.42)$ than low communion $(M=4.12, S D=1.93)$, $F(1,61)=11.26, p=.001, \eta_{\mathrm{p}}{ }^{2}=.16[.03, .32]$; for the senders low in agency, the effect of communion on happiness contagion was not significant (high communion: $M=4.97$, 
Panel A

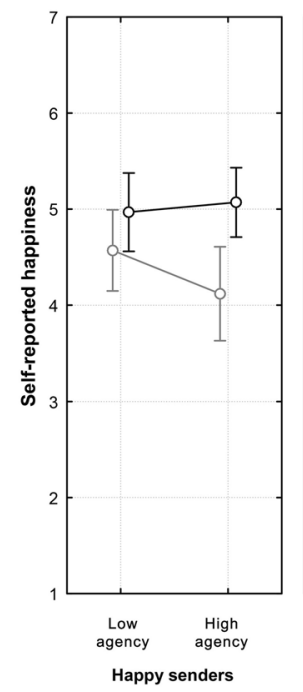

Panel B

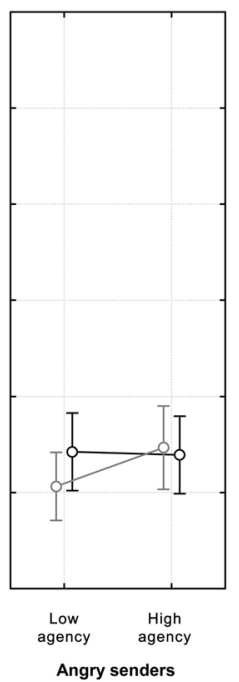

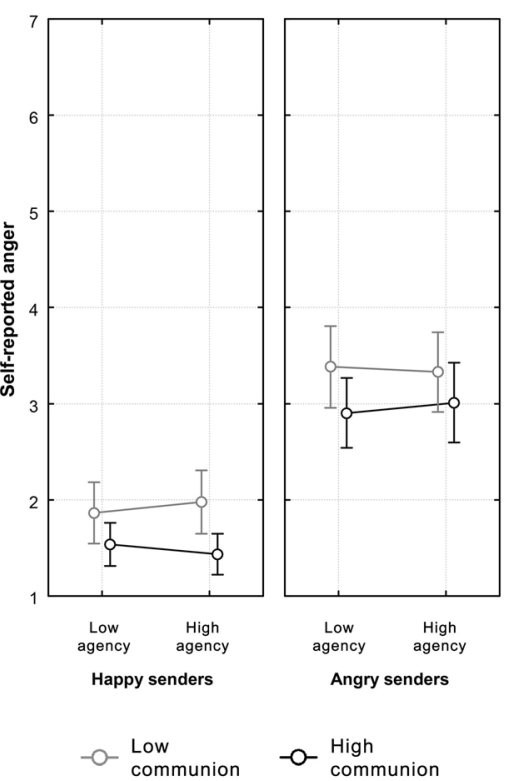

Panel C

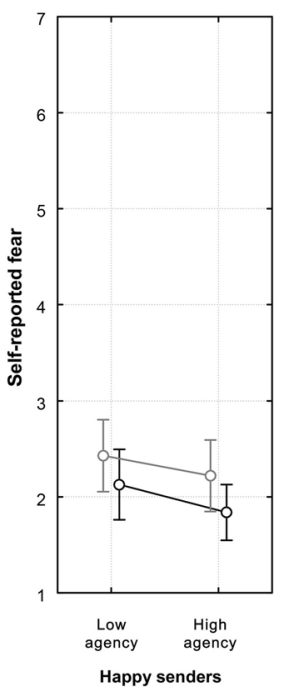

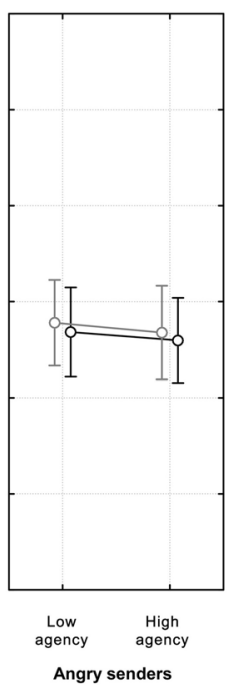

scores indicating higher levels of self-reported emotions. Error bars represent $95 \%$ confidence intervals

senders than high-communion senders), but these responses were stronger when additional information about the senders' low agency was given.

The analyses for self-reported fear also support the idea that anger evoked reactive (rather than imitative) responses (Fig. 3, Panel C). Specifically, participants reported higher levels of fear after exposure to anger displays $(M=3.68$, $S D=1.49)$ than after exposure to happiness displays $(M=2.15, S D=0.91)$, as indicated by the large effect size of this comparison $\left(\eta_{\mathrm{p}}{ }^{2}=.56[.39, .67]\right)$. All other contrasts as well as the three-way interaction were small $\left(\eta_{\mathrm{p}}{ }^{2} \leq .03\right)$.

Summing up, Study 2 provided further evidence that agency and communion exerted interactive effects on happiness contagion and these effects aligned with out predictions. When it comes to the induction of anger, the results of Study 2 mirrored those of Study 1 to a limited extent. Specifically, it turned out that more convergent responses to anger occurred when the senders were low in communion than when they were high in communion. This shows that, similar to Study 1, participants were more angry when anger was displayed by the senders who had non-affiliative traits, which suggests that participants' convergent responses to anger qualified as reactive rather than imitative responses (Fischer \& Hess, 2017). This time, however, convergent responses to anger were stronger when agency was low, so the senders were less able to act on their intentions. This discrepancy between Studies 1 and 2 is surprising, given that the procedures of both studies were very similar. The only difference that could have contributed to the differential 
patterns of results across the two studies was the number of emotional displays participants were exposed to. Possibly the removal of sadness, that is, a display that shares some features with both happiness (i.e., intrinsic affiliative meaning) and anger (i.e., negative valence), highlighted the difference between happiness and anger but it is difficult to explain why this difference might have reversed the role of agency. Therefore, the role od agency in the induction angerrelated responses warrants further examination.

\section{Emotional mimicry}

Our analytic strategy for emotional mimicry consisted of two steps. Initially, as stated in the preregistration, we focused on separate Action Units (AUs). The results of this analysis, however, turned out to be quite complex and, except for AU4 (initially treated as indicative of anger), showed no effects of the senders' emotional displays on participants' emotional expression (see Supplementary Material for details). We should note, however, that null effects of emotional displays on participants' responses are not uncommon when FACScoding is used (e.g., Sachisthal et al., 2016), which may be attributed to the fact that facial expressions involve patterns of muscle activity rather than separate AUs (Cohn \& Ekman, 2005; Friesen \& Ekman, 1983). Therefore, in addition to our pre-registered analyses, we run complementary analyses based on the recommendations that mimicry needs to capture the joint movement of the muscles involved in a given facial expression rather than be analyzed on a muscle by muscle basis (Hess et al., 2017; Olszanowski et al., 2020). These recommendations go beyond mimicry assessments and can be applied to measuring facial activity in general (e.g., emotional reactions to pictorial stimuli; Hess et al., 2017). Accordingly, we calculated a facial activity index by subtracting the intensity of AU4 from the intensity of AU12 for happiness and the intensity of AU12 from the intensity of AU4 for anger. Higher scores indicated more intense mimicry.

We then performed a 2 (senders' emotional display: happiness, anger) $\times 2$ (senders' communal traits: high communion, low communion) $\times 2$ (senders' agentic traits: high agency, low agency) repeated measures ANOVA, with facial activity index as a dependent variable, followed by a series of planned contrasts. The predicted three-way interaction did not near significance, $F(1,58)=.398 ; p=.531$; $\eta_{\mathrm{p}}{ }^{2}=.01[0, .10]$ (see Fig. 4). The first contrast indicated that the index was higher (i.e., mimicry more intense) when participants were exposed to the happy senders $(M=0.39$, $S D=0.84)$ than when they were exposed to the angry senders $(M=-0.07, S D=1.06), F(1,58)=4.93 ; p=.030$; $\eta_{\mathrm{p}}{ }^{2}=.08[0, .23]$. The second contrast showed that happiness mimicry was stronger when the happy senders were high in communion $(M=0.57, S D=0.93)$ than low in communion

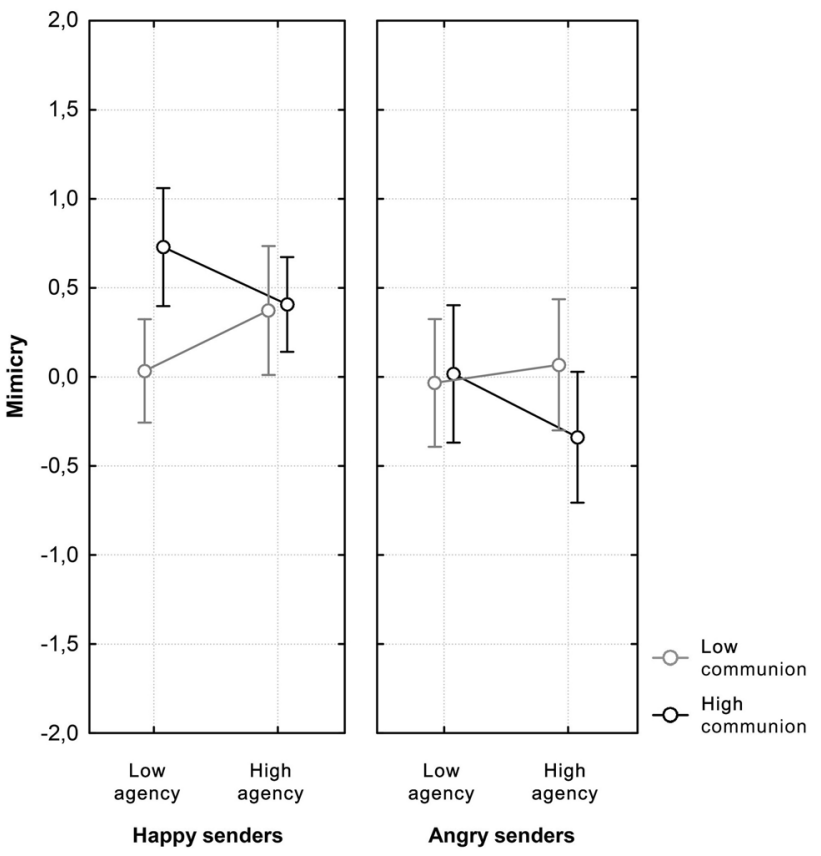

Fig. 4 Facial activity index (mimicry) as a function of the senders' communal traits, agentic traits and emotional expression (Study 2). Scores range from -3 to 3 , with higher scores indicating more intense mimicry. Error bars represent $95 \%$ confidence intervals

$(M=0.20, S D=1.00), F(1,58)=8.92 ; p=.004 ; \eta_{\mathrm{p}}{ }^{2}=.13$ $[.01, .29]$. Finally, the last two contrasts indicated that for the senders low in agency, communion influenced happiness mimicry, such that high communion was associated with more happiness $(M=0.73, S D=1.27)$ than low communion $(M=0.03, S D=1.11), F(1,58)=16.44, p<.001, \eta_{\mathrm{p}}{ }^{2}=.22$ $[.06, .39]$; for the senders high in agency, the effect of communion on happiness mimicry was not observed (high communion: $M=0.41, S D=1.02$ vs. low communion: $M=0.37$, $S D=1.39), F(1,58)=0.04, p=.840, \eta_{\mathrm{p}}^{2}=.001[0, .06]$.

Taken together, the effects observed for self-reported emotions were reflected by participants' facial activity only to a limited extent. Specifically, in line with recent models of emotional mimicry, which hold that intrinsically affiliative emotional expressions are more likely to evoke imitative responses than intrinsically non-affiliative emotional expressions (see Hess \& Fischer, 2014), we found that happiness was imitated to a higher extent than anger. Moreover, happiness mimicry was strengthened by high communion. This positive effect of communion, however, was more pronounced when the senders were low in agency. Thus, contrary to our predictions and the assumptions of the Dual Perspective Model of Agency and Communion, the knowledge that the senders were able to act on their affiliative intentions cushioned (rather than strengthened) the effect of the senders' affiliative intents on happiness mimicry. This result may mean that the role of agency in happiness 
mimicry is the opposite of its role in happiness contagion. However, it is more likely that this surprising finding may be due to the fact that, similar to other studies that relied on FACS-coding (e.g., Sachisthal et al., 2016), the number of zeros (indicating no occurrence of a given AU) in our study was very high. This suggests that participants' subtle facial muscle activity was not visible to the naked eye and the data obtained with FACS-coding should be treated with caution. Moreover, as presented in Supplementary Table S3, we found no significant correlations between self-reported emotions and facial activity (except for negative correlations between self-reported anger and AUs 6 and 12). Although, as already mentioned, discrepancies between mimicry and contagion have already been reported (Hess \& Blairy, 2001; Van der Schalk et al., 2011), it is possible that our measure of facial activity was not sensitive enough. We addressed this issue in Study 3, using a more valid method for measuring momentary facial movement.

\section{Study 3}

Study 3 was motivated by the inconclusive findings of Studies 1 and 2. Additionally, our aim was to replicate the effects of agency and communion on happiness contagion we consistently observed across both previous studies. In Study 3 , we used three emotional displays again and measured participants' facial activity using electromyography (EMG). EMG captures facial activity that is barely visible or invisible to the naked eye and thus outperforms FACScoding when measuring very subtle responses such as facial mimicry (Hess, 2021).

Initially, we were also planning to use FACS-coding, but the settings of the software we used made it impossible to record videos during the study. Specifically, iMotion 7.0 that recorded both camera input and the screen stopped the recording process the moment stimuli presentation was switched to the full-screen mode. This technical error was detected after the data collection was completed. Therefore, contrary to the preregistered protocol, FACS-coding was not performed.

\section{Method}

\section{Participants and design}

We recruited 73 university students but two participants who guessed the hypotheses were excluded from the analyses. The final sample comprised 71 participants (62 women; $M_{\text {age }}=25.89$ years, $S D=7.27$ ). Moreover, we could not use the EMG recordings of 7 participants because of technical problems and artifacts in the signal. Thus, the EMG analyses were based on 64 participants (55 women). Similar to Study
1, we used a 3 (senders' emotional display: happiness, anger, sadness) $\times 2$ (senders' communal traits: high communion, low communion) $\times 2$ (senders' agentic traits: high agency, low agency) within-participants design.

\section{Procedure and materials}

The procedure of Study 3 was similar to that of Study 1 except that this time, to minimize the risk that participants would not watch twelve videos carefully, we explicitly asked them to do so. Moreover, we measured the activity of the corrugator supercilii (which lowers the eyebrows), zygomaticus major (which pulls up lip corners), and depressor anguli oris (which lowers lip corners) by the bipolar placement of surface, $8 \mathrm{~mm}$ diameter, $\mathrm{Ag} / \mathrm{Cl}$ electrodes on the left side of the face. A ground electrode was attached to the middle of the forehead, directly below the hairline (Tassinary et al., 2007). EMG was measured using a BioPac MP150, digitized with 24-bit resolution, sampled at $1 \mathrm{kHz}$, and recorded on a PC. Raw data were filtered offline with a $20-400 \mathrm{~Hz}$ bandpass filter and a $50-\mathrm{Hz}$ notch filter and rectified using the ANSLAB software (Blechert et al., 2016). The signal was baseline corrected (i.e., we calculated muscle activity by subtracting the baseline activity from the average of all data points in each trial). The data were standardized for each participant separately. First, they were averaged within $500 \mathrm{~ms}$ epochs across a trial (i.e., $35 \mathrm{~s}$ ), which resulted in 70 data points for each trial, and the baseline including $1 \mathrm{~s}$ prior to the stimuli onset. Next, the data were standardized within each participant and within each muscle using mean and standard deviation computed from all collected data points across the study $(12$ trials $\times 71$ data points $=852)$. All data points above three standard deviations were removed (1.8\% of all data points). We also removed the trials containing more than 15 such data points ( $2.6 \%$ of all trials). ${ }^{5}$

Participants also rated the degree to which they felt happiness $(\omega=.93)$, sadness $(\omega=.86)$, anger $(\omega=.88)$, and fear $(\omega=.86)$ using the modified DES (Izard et al., 1974) and completed two recall tasks rating the senders' communion $\left(r_{S B \text { fist task }}=.93 ; r_{S B \text { second task }}=.75\right)$ and agency $\left(r_{S B \text { first task }}=.81 ; r_{S B \text { second task }}=.74\right)$.

\footnotetext{
5 As due to due to technical error, we did not have the recordings from Study 3, we were unable to remove the trails in which participants demonstrated some gross behaviors that might have had an impact on the signal (e.g., yawning or touching the electrodes). Nevertheless, we assume that in most cases the artifacts caused by gross behaviors were removed because they manifested as outliers. Such behaviors typically cause considerable changes in the signal and therefore, when all data points above three standard deviations were removed, these artifacts were also eliminated.
} 


\section{Results}

\section{Manipulation check}

Planned contrast indicated that participants rated the high-communion senders as more communal $(M=5.91$, $S D=0.94)$ than the low-communion senders $(M=1.78$, $S D=0.81), F(1,70)=507.55, p<.001, \eta_{\mathrm{p}}{ }^{2}=.88[.82, .91]$, and the high-agency senders as more agentic $M=6.16$, $S D=0.78)$ than the low-agency senders $(M=2.12$, $S D=0.93), F(1,70)=499.43, p<.001, \eta_{\mathrm{p}}{ }^{2}=.88[.82, .91]$. All means and standard deviations are given in Supplementary Table S7. Taken together, these findings show that our trait manipulation was successful.

\section{Emotional contagion}

We first performed a 3 (senders' emotional display: happiness, sadness, anger) $\times 2$ (senders' communal traits: high communion, low communion) $\times 2$ (senders' agentic traits: high agency, low agency) repeated measures ANOVA, with self-reported happiness as a dependent variable. The analysis showed a significant three-way interaction, $F(2,140)=3.08$, $p=.049, \eta_{\mathrm{p}}{ }^{2}=.04[0, .11]$ (Fig. 5, Panel A). The first contrast was significant, $F(1,70)=128.80, p<.001, \eta_{\mathrm{p}}{ }^{2}=.65[.51$, 73]. Participants reported being more happy after seeing the happy senders $(M=4.44, S D=1.42)$ than after seeing the angry and sad senders $(M=2.15, S D=1.02)$. Moreover, as evidenced by the second contrast, participants reported being more happy after seeing the happy high-communion senders $(M=4.58, S D=1.47)$ than the happy low-communion senders $(M=4.31, S D=1.55), F(1,70)=5.44, p=.025, \eta_{\mathrm{p}}{ }^{2}=.07$ $[.001, .21]$. Finally, we observed, that the effect of communion on happiness contagion was present when the senders were high in agency (high communion: $M=4.66, S D=1.74$; low communion: $M=4.11, S D=1.95), F(1,70)=7.19$, $p=.009, \eta_{\mathrm{p}}{ }^{2}=.09[.01, .23]$, while for the senders low in agency, communion did not affect happiness contagion (high communion: $M=4.50, S D=1.68$; low communion: $4.50, S D=1.68), F(1,70)<0.01, p=.979, \eta_{\mathrm{p}}^{2}<.01[0, .03]$. Altogether, high agency fostered the effects of high communion on participants' self-reported happiness, whereas low agency lessened these effects, thereby replicating the interactive effects of both social dimensions on happiness contagion we already observed in Studies 1 and 2.

In a similar analysis for self-reported sadness, we found no support for the predicted three-way interaction, $F(2,140)=1.30 ; p=.274 ; \eta_{\mathrm{p}}{ }^{2}=.02[0, .07]$ (see Fig. 5, Panel B). Only the first contrast was significant indicating that participants reported being sadder following exposure to sadness displays $(M=3.46, S D=1.51)$ than happy and angry displays $(M=1.88, S D=0.73), F(1,70)=82.90$; $p<.001 ; \eta_{\mathrm{p}}{ }^{2}=.54[.38, .65]$. The remaining contrasts testing the role of agency and communion on sadness contagion were not significant $(F \mathrm{~s}<3.30, p \mathrm{~s}>0.073)$. This again shows that sadness contagion occurred irrespective of the senders' traits. As already mentioned, this result does not align with our hypotheses but corresponds to other studies showing that sadness may be shared regardless of the senders' affiliative vs. not-affiliative characteristics (e.g., Wróbel \& Królewiak, 2017; Wróbel et al., 2020). We return to this point in the General Discussion.

Next, similar to Studies 1 and 2, we analyzed participants' responses to anger focusing on self-reported anger and fear. For self-reported anger, the analysis showed a small-sized three-way interaction $\left(\eta_{\mathrm{p}}{ }^{2}=.05[0, .13]\right)$ (Fig. 5, Panel C). Generally, participants reported higher levels of anger following exposure to anger displays $(M=2.64, S D=1.42)$ than happiness and sadness displays $(M=1.77, S D=0.85)$, as evidenced by a large effect size of this comparison $\left(\eta_{\mathrm{p}}{ }^{2}=.28[.11, .43]\right)$. We also found that high communion was associated with slightly less anger in response to angry senders $(M=2.54, S D=1.58)$ than low communion $(M=2.73, S D=1.47)$, but the effect size of this comparison was small $\left(\eta_{\mathrm{p}}{ }^{2}=.03[0, .13]\right)$. Finally, the effect of communion on self-reported anger was more pronounced for the senders low in agency, such that high communion was associated with less anger $(M=2.54$, $S D=1.84)$ than low communion $(M=2.93, S D=1.77)$, $\eta_{\mathrm{p}}{ }^{2}=.06[0, .18]$; for the senders high in agency, the effect of communion on self-reported anger was negligible (high communion: $M=M=2.55, S D=1.75$ vs. low communion: $M=2.53, S D=1.67), \eta_{\mathrm{p}}{ }^{2}<.01[0, .04]$. These results indicate that the two dimensions jointly modulated responses to anger. In general, more convergent responses to anger were fostered by low communion rather than high communion, which again shows that anger displays evoked reactive rather than imitative responses. Yet, across the three studies, agency modulated these responses in an inconsistent way. We elaborate on these inconsistencies in the General Discussion.

Finally, the analyses for self-reported fear showed that anger evoked complementary reactive responses (Fig. 5, Panel D). Specifically, participants reported higher levels of fear after exposure to anger displays $(M=2.76, S D=1.33)$ than happiness and sadness displays $(M=1.97, S D=0.81)$, as indicated by the large effect size of this comparison $\left(\eta_{\mathrm{p}}{ }^{2}=.38[.21, .52]\right)$. All other contrasts as well as the threeway interaction were small $\left(\eta_{\mathrm{p}}{ }^{2} \leq .02\right)$.

Summing up, the results of Study 3 were in line with our hypotheses and the assumptions of the Dual Perspective Model of Agency and Communion (Abele \& Wojciszke, 2014) only for happiness contagion (while for sadness contagion we found no effects of the senders' traits). The pattern we observed for self-reported anger suggested that angry expressions evoked convergent and complementary reactive 
Panel A

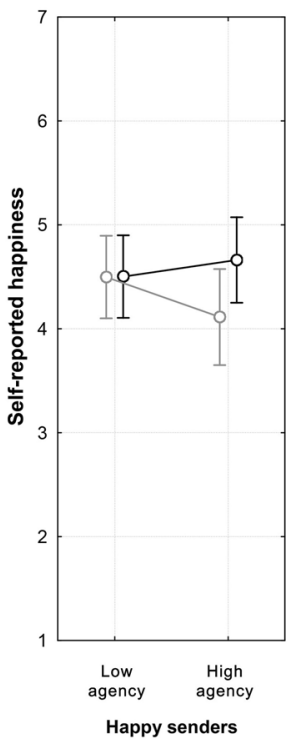

Panel C

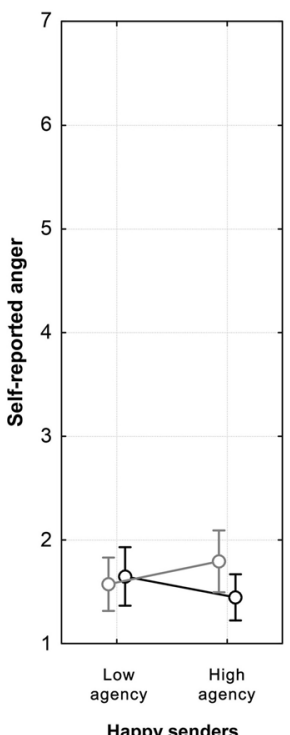

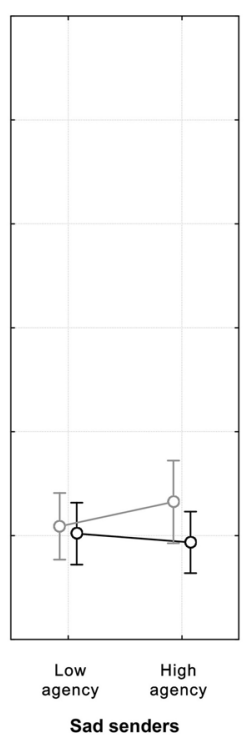

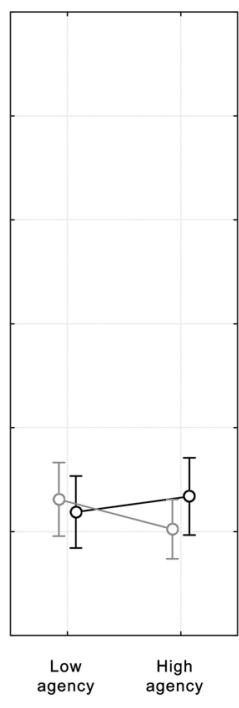

Angry senders

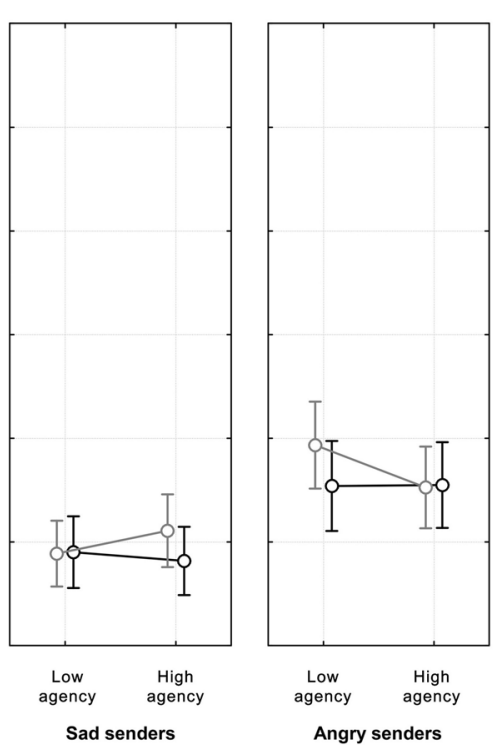

$-0-$ Low

Fig. 5 Participants' self-reported happiness (A), sadness (B), anger (C), and fear (D) as a function of the senders' communal traits, agentic traits and emotional expression (Study 3). Scores range from 1 to

responses (albeit complementary fear in response to anger occurred irrespective of the senders' traits).

\section{Emotional mimicry}

Following the assumption that mimicry cannot be identified by the activity of single muscles (Hess et al., 2017;

\section{Panel B}
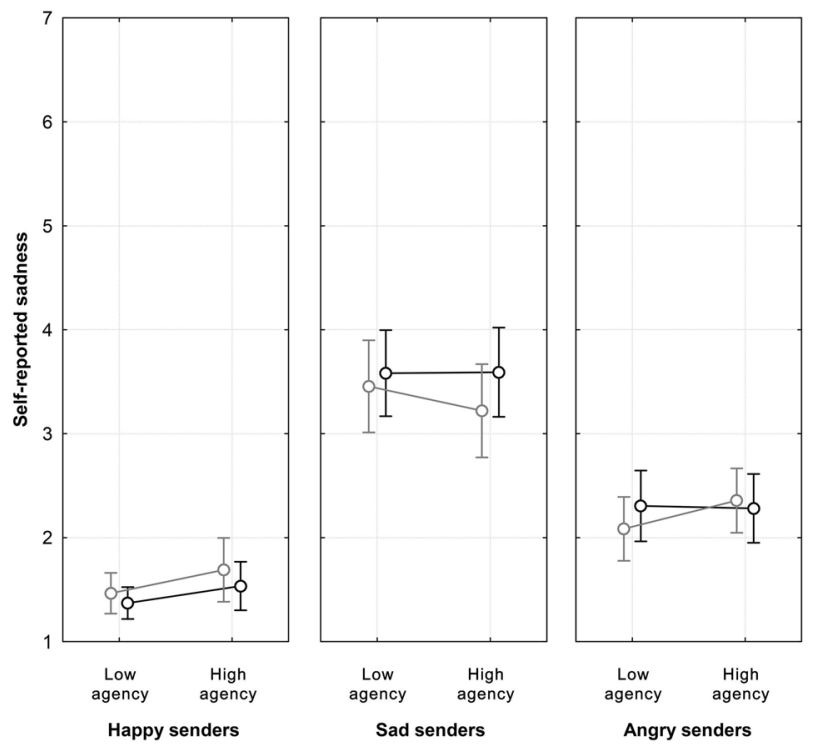

\section{Panel D}
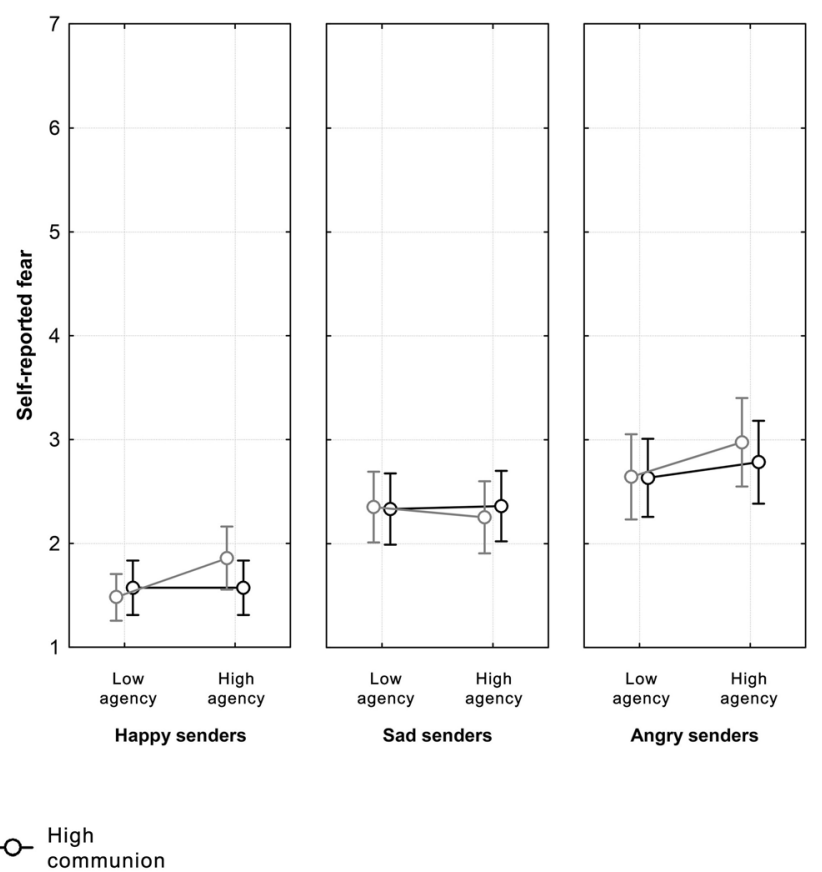

7, with higher scores indicating higher levels of self-reported emotions. Error bars represent $95 \%$ confidence intervals

Olszanowski et al., 2020), mimicry to happy displays was calculated by subtracting the activity of corrugator supercilii from the activity of zygomaticus major, while mimicry for angry and sad displays was calculated by subtracting the activity of zygomaticus major from the activity of corrugator supercilli (thus, the index was analogical to that used in Study 2). Higher scores indicated more intense mimicry. We 
Fig. 6 Facial activity index (mimicry) as a function of the senders' communal traits, agentic traits and emotional expression (Study 3). Higher scores indicate more intense mimicry. Error bars represent $95 \%$ confidence intervals
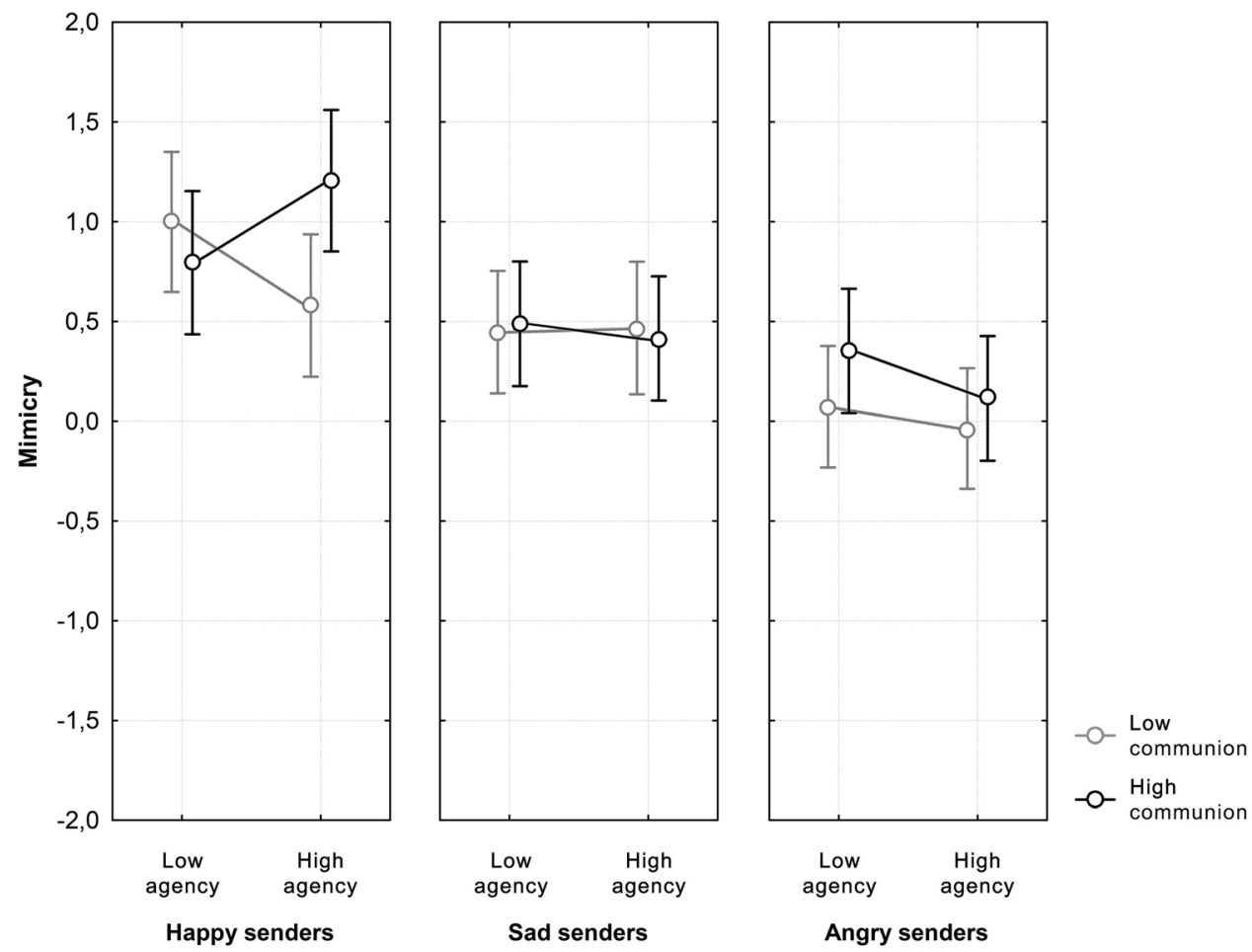

also planned to calculate an additional facial activity index for sadness by subtracting the activity of depressor from the activity of zygomaticus major, but there was cross-talk between zygomaticus major and depressor, as indicated by the positive correlation between the two (see Supplementary Table S6). Thus, we had to abandon this preregistered idea.

As we removed artifacts from the signal and thus some cases were incomplete, we used multilevel modelling (MLM) with maximum likelihood to deal with missing data and obtain unbiased estimates for means of muscle activity. MLM is based on maximum likelihood estimation that uses all of the available data to generate parameter estimates without excluding incomplete cases from the analysis (Enders, 2011). The strategy of model building was based on a priori specification of fixed effect structure that included the senders' emotional display (happiness, anger, sadnessdifference-coded with happiness as the contrast condition to which anger and sadness levels were compared), communal traits (high communion, low communion), and agentic traits (high agency, low agency). Next, we built random effect structure, starting with the simplest structure that included only intercept fit across participants, and then adding random effects of each factor (random slopes) along with their interactions. Additionally, as preliminary analysis revealed that mimicry tended to increase after the initial trials, we also tested the models with the trial number and its quadratic term (z-standardized, i.e., centered) added as a covariate. The final model was selected based on the lowest value of Akaike Information Criterion (AIC), with random effect structure that, in addition to a random intercept, included random effects of emotional display, and the covariance structure that was set as correlated (unstructured).

The results showed the expected three-way interaction, $B=.938[0.28,1.60], t(623.6)=2.79, p=.005$ (Fig. 6). The main effect of emotional display that contrasted happiness display with two other emotional displays was also significant, $B=.606[0.29,0.93], t(64)=3.71, p<.001$. Specifically, mimicry was more intense after exposure to the happy senders $(M=0.89 ; S E=0.11)$ than after exposure to the sad and angry senders $(M=0.45, S E=0.11$ and $M=0.12$, $S E=0.11$, respectively). Next, we performed a series of simple effect analyses consistent with our hypotheses. We found that happiness mimicry was not moderated by the senders' communion (high communion: $M=1.00, S E=0.15$ vs. low communion: $M=0.79, S E=0.15), B=.216$ [ $-0.06,0.49$ ], $t(624)=1.56, p=.119$. However, we found that for the highagency senders, communion affected happiness mimicry, such the high-communion senders were mimicked more $(M=1.21, S E=0.18)$ than the low-communion senders $(M=0.57, S E=0.18), B=.63[0.25,1.01], t(624)=13.25$, $p<.001$; for the senders low in agency, communion did not influence happiness mimicry (high communion: $M=0.80$, $S E=0.18$ vs. low communion: $M=1.00, S E=0.18), B=.199$ $[-0.19,0.58], t(625)=1.01, p=.311$.

Overall, the analysis of participants' facial activity as measured with EMG provided additional support for the notion that agency and communion modulated participants' responses to happiness displays in line with the Dual Perspective Model 
Table 2 Meta-analysis of the effects of emotional display, communion and agency on self-reported happiness (Studies 1, 2, and 3), sadness (Studies 1 and 3), and anger (Studies 1, 2, and 3)

\begin{tabular}{|c|c|c|c|c|c|c|c|c|}
\hline \multirow[t]{2}{*}{ Self-reported emotion } & \multicolumn{2}{|c|}{$\begin{array}{l}\text { Contrast } 1 \text { : target emotion } \\
\text { vs. other emotions }\end{array}$} & \multicolumn{2}{|c|}{$\begin{array}{l}\text { Contrast 2: low vs. high } \\
\text { communion }\end{array}$} & \multicolumn{2}{|c|}{$\begin{array}{l}\text { Contrast } 3 \text { : low vs. high com- } \\
\text { munion plus high agency }\end{array}$} & \multicolumn{2}{|c|}{$\begin{array}{l}\text { Contrast } 4 \text { : low vs. high } \\
\text { communion plus low } \\
\text { agency }\end{array}$} \\
\hline & $d[95 \% \mathrm{CI}]$ & $p$ & $d[95 \% \mathrm{CI}]$ & $p$ & $d[95 \% \mathrm{CI}]$ & $p$ & $d[95 \% \mathrm{CI}]$ & $p$ \\
\hline Happiness & $2.12[1.65,2.58]$ & $<.001$ & $0.33[0.12,0.53]$ & .002 & $0.44[0.19,0.68]$ & $<.001$ & $0.12[-0.11,0.35]$ & .305 \\
\hline Sadness & $1.43[1.00,1.86]$ & $<.001$ & $0.08[-0.20,0.37]$ & .559 & $0.12[-0.20,0.44]$ & .464 & $0.05[-0.27,0.36]$ & .770 \\
\hline Anger & $1.10[0.71,1.47]$ & $<.001$ & $-0.20[-0.41,0.01]$ & .061 & $-0.17[-0.41,0.06]$ & .153 & $-0.20[-0.44,0.04]$ & .102 \\
\hline
\end{tabular}

of Agency and Communion. At the same time, we observed that sadness and anger were mimicked less than happiness displays, which possibly resulted from the fact that people, in general, are more eager to imitate happiness than other emotional displays (Hess \& Fischer, 2014). This may be due to the fact that although sad faces are assessed as more affiliative than angry faces, they are still less affiliative than happy faces (Hess et al., 2000).

Another important finding is that, even though agency and communion moderated happiness contagion and mimicry in a comparable way, we observed no relationship between the two processes (see Supplementary Table S6). Although no support for the mimicry-contagion link has already been reported in the literature (e.g., Hess \& Blairy, 2001; Van der Schalk et al., 2011), a previous study using the same videos we used in the current study found such support (Olszanowski et al., 2020). One possible reason for this positive finding is that this study was conducted in acontextual settings (no information about the sender was provided), whereas our study, similar to other research (Hess \& Blairy, 2001; Van der Schalk et al., 2011), included additional information about the senders. Possibly when no contextual information is given, the engagement of additional more controlled processes in emotional contagion is limited and thus, the relation between mimicry and contagion is easier to observe (see also Wróbel \& Imbir, 2019). This suggests that, despite the fact that social factors may influence emotional mimicry and contagion similarly, different conceptualizations and operationalizations of the two processes (i.e., facial activity and self-reported emotions, respectively) may contribute to an inconsistent pattern of findings regarding the relationship between them. We should also stress that, in general, experiential, behavioral, and physiological measures of emotion are not always directly related (Fridlund, 1994; Mauss \& Robinson, 2009; Parkinson, 2005), which adds to the complexity of this pattern.

\section{Meta-analysis of the effects of agency and communion on self-reported emotions}

To estimate the effects of the senders' emotional displays, communion and agency on emotional contagion across the three studies we ran a series of meta-analyses (see Table 2). The analyses were carried out using the effect size and effect size standard error. Models were estimated using restricted maximum-likelihood. The results showed that participants' self-reported emotions were congruent with the senders' displays but the effects of agency and communion on these congruent responses varied across the three studies. Specifically, for self-reported happiness, we found that high communion promoted congruent responses to the senders' expressed happiness, but this effect was present only when the senders were high in agency; for the senders low in agency, communion did not affect happiness contagion. For self-reported sadness, the effects of the senders' communal and agentic traits were negligible. Finally, for self-reported anger, we found that congruent responses were promoted by low rather than high communion but no evidence for the modulating role of agency was observed.

\section{General discussion}

The current research addressed the role of the two fundamental dimensions of social perception in emotional contagion and mimicry. Drawing upon previous research showing that emotions are more likely to by shared and imitated in affiliative than in non-affiliative social contexts (for a review, see Fischer \& Hess, 2017), we expected that 
high communion would foster the induction of concordant responses to intrinsically affiliative emotional displays, that is, happiness and sadness, whereas low communion would hinder such responses. Moreover, based on the Dual Perspective Model of Agency and Communion (Abele \& Wojciszke, 2014), we hypothesized that the effects of communion on participants' emotional responses to happiness and sadness would be strengthened by the senders' high agency. We did not formulate any specific predictions concerning an intrinsically non-affiliative emotional display, that is, anger.

In line with our hypotheses, the findings for self-reported happiness consistently showed that when the senders expressed happiness, agency and communion jointly modulated participants' responses. In general, happiness contagion was more pronounced after exposure to the happy senders high in communion than the happy senders low in communion, but only when additional information about the senders' high agency was given. When the senders were low in agency, the effect of communion on self-reported happiness following exposure to the happy senders was not observed in any of the three studies. Furthermore, these interactive effects of agency and communion were mirrored by similar effects on happiness mimicry, as measured with EMG (Study 3). Our findings are thus consistent with recent views on emotional mimicry and contagion (Fischer \& Hess, 2017; Hess, 2021; Wróbel \& Imbir, 2019) as well as the Dual Perspective Model of Agency and Communion (Abele $\&$ Wojciszke, 2014). We believe that combining these theoretical approaches provides a fresh view on emotional convergence between people by showing that the top-down social control of happiness contagion and mimicry may be explained by the rules already established in the social cognition domain. Specifically, focusing on both dimensions of social cognition enabled us to demonstrate that the sender's communal (i.e., affiliative) characteristics are an important moderator of happiness contagion and mimicry, but low agency (that is, the information that the sender is unable to enact his/her intents) may eliminate this moderating effect.

The question remains, though, why the modulating effect of agency on happiness mimicry was reversed in Study 2 that relied on FACS-coding. Here, happiness mimicry was fostered by high communion only when the senders were low agency. This result is opposite to the effects of agency we consistently observed for happiness contagion (i.e., selfreported happiness) and happiness mimicry (as measured with EMG). On the one hand, these discrepancies seem surprising, given that FACS-coding correlates with EMG scores (Cohn \& Ekman, 2005) and is characterized by high reliability (Sayette et al., 2001). On the other hand, although FACS-coding is often used to measure facial activity, including facial mimicry (Sachisthal et al., 2016; van der Schalk et al., 2011), it may be difficult to implement when facial activity is barely visible or invisible (Hess, 2021; van Boxtel, 2010). Therefore, although EMG has some limitations that FACS-coding does not have (e.g., the number of muscle sites that the electrodes can be attached to is limited or the reliable differentiation between specific emotions may be difficult due to cross-talk), EMG is better-suited for the assessment of subtle facial activity such as emotional mimicry (Hess, 2021). As a result, it is possible that FACS-coding enabled us to show that communion strengthened happiness mimicry, but the differences in facial activity resulting from the impact of agency were invisible even to the trained FACS-coders.

The findings for self-reported sadness showed that sadness contagion occurred regardless of the senders' traits. This result is contrary to our reasoning because sadness is an intrinsically affiliative emotional display and thus, we expected that sadness contagion (similar to happiness contagion) would be fostered by a combination of high communion and high agency. Yet, the fact that participants shared the senders' sadness irrespective of these senders' traits aligns with other studies that found no effects of the senders' characteristics on participants' responses to sadness (e.g., Weyers et al., 2009; Wróbel et al., 2020). For instance, Wróbel and Królewiak (2017) demonstrated that the tendency to share the sender's sadness did not disappear even when the sender was presented in a highly unfavorable light. In a similar vein, Weyers et al. (2009) demonstrated that facial reactions to expressed sadness did not differ between competition primed participants and neutrally primed participants, even though the former perceived the senders as less likeable than the latter. One possible explanation is that responses to sadness reflect emotional aspects of empathy. Accordingly, participants might have felt compassion towards the sad senders regardless of whether these senders had affiliative or non-affiliative traits. For instance, similar effects were found for emotional responses to tears (i.e., participants reacted similarly to crying ingroup and outgroup members; Balsters, 2015; Bobowik et al., 2020). Alternatively, as the expressed emotion may override the information about the sender's characteristics (e.g., Olszanowski et al., 2019), it is possible that participants did not pay attention to the sad senders' profiles. This, as already noted, may apply to sadness in particular, because sadness communicates helplessness and triggers the intention to offer support (Fischer \& Manstead, 2008; Van Kleef et al., 2011), which may divert the receivers' attention from the senders' traits and, in consequence, eliminate the effects of these traits on sadness contagion.

A fairly complex pattern of results was also found for anger. In general, in all three studies, participants reacted with more anger to the low-communion angry senders than to the high-communion angry senders, which shows that anger displays evoked more anger when the senders' intents were non-affiliative. This suggests that anger displays 
probably elicited reactive rather than imitative responses (Fischer \& Hess, 2017). Such reactive anger occurs when angry expressions are perceived as threatening (which in the case of expressed anger happens very often-for instance anger often evokes a significantly heightened startle response; Springer et al., 2007). In line with this reasoning, expressed anger also evoked complementary reactive fear (even though fear occurred regardless of the senders' traits). The role of agency in the induction of reactive anger, however, varied across the three studies: In Study 1, the effect of communion was more pronounced for the high agency senders, whereas in Studies 2 and 3, this effect was more pronounced for the low agency senders. The results of Studies 2 and 3 thus seem counterintuitive because one could expect that reactive anger should occur particularly in response to the senders characterized by low communion combined with high agency, because such senders seem particularly threatening (i.e., they are more able to act on their non-affiliative intentions than low-agency senders). Moreover, people characterized by low communion and high agency (even when portrayed with no emotional display) often elicit anger in others (Fiske et al., 2002) and anger expressed by high-agency people is easier to notice than anger expressed by low-agency people (Ratcliff et al., 2012). However, as mentioned in the Introduction, previous research on the role of the senders' characteristics in modulating the receivers' responses to anger is also inconclusive (van der Schalk et al., 2011; Weisbuch \& Ambady, 2008) and much empirical evidence shows that people often avoid imitating anger (Fischer \& Hess, 2017). Hence, it is possible that emotional responses to intrinsically non-affiliative displays such as anger depend on a more complex set of factors than responses to intrinsically affiliative displays and thus are less predictable.

Limitations of this study should be noted. First, we relied on "contagious" material of known effectiveness to diminish the impact of confounding variables. However, the extent to which morphed videos simulate real emotional displays is limited. Thus, even though naïve viewers who watched the videos during the preliminary study, perceived them as dynamic expressions (Olszanowski et al., 2020) and congruent emotional responses have been shown to be elicited not only by real faces but also by avatars (e.g., Weyers et al., 2009), future research would benefit from examining the effects of agency and communion on emotional contagion and mimicry in more realistic interactions. Moreover, using morphed videos made it difficult to include a control condition, because the videos showed transformations from a neutral face to a full emotional display and thus, creating an adequate video with a neutral expression was impossible. As a result, it is difficult to assess whether the scores reflected an increase, a decrease or no change in participants' emotional responses, which makes the interpretation of the effects of the senders' traits on these responses less straightforward. Second, although previous research on gender effects in emotional contagion and mimicry is highly inconclusive (for a review, see Lehane, 2015), we did not test any gender effects in the current study because the samples were too small and the number of men in these samples was low. However, given that some studies suggest that sensitivity to social and emotional information may differ across genders (for a review, see Fischer et al., 2018), testing the role of the receivers gender in emotional contagion and mimicry would be an important extension of the current findings. Third, the measures we used to assess emotional mimicry turned out to be difficult to compare due to the technical error in Study 3. Hence, future research might assess facial activity with complementary measures within one study to make comparisons between different measures more straightforward. Finally, based on previous studies (e.g., Hareli \& Hess, 2012), we assumed that the effects of agency and communion on participants' responses would be driven by their influence on participants' evaluations of the senders' intents. Yet, as the procedure of all studies was long, we did not measure these evaluations directly, so all conclusions referring to the senders' intents should be treated with caution. Future research should be supplemented with such measures.

Overall, our research contributes to the literature by showing that the interplay of agency and communion plays an important role in emotional contagion and mimicry. This role, as suggested by our findings, may change depending on the emotion displayed by the sender. Therefore, the results of our studies align with the notion that the sender's characteristics and emotions he/she expresses jointly contribute to the general evaluation of the sender and thus interact when activating top-down mechanisms controlling emotional contagion (Fischer \& Hess, 2017; Wróbel \& Imbir, 2019). Of importance, these mechanisms, as shown by our findings for happiness contagion, seem highly sophisticated because they may be activated by the information on not only whether the sender has affiliative intents (i.e., communion) but also on whether he/she is able to enact these intents (i.e., agency). Our studies thus extend the assumptions of the Dual Perspective Model of Agency and Communion (Abele \& Wojciszke, 2014) to a "new" class of phenomena, namely emotional contagion and mimicry. These assumptions proved particularly valid when the senders expressed happiness, that is, an emotion that is most readily shared and imitated (Fischer \& Hess, 2017). The limited applicability of the Dual Perspective Model of Agency and Communion to participants' responses to sadness and anger points to the need to further investigate moderators of emotional contagion and mimicry when other emotions are displayed by the sender. We hope that future studies will address this issue. 
Supplementary Information The online version contains supplementary material available at https://doi.org/10.1007/s11031-021-09897-z.

Acknowledgements We thank E. Bzikowska, P. Głowacka, Z. Górska, N. Kosztowny, E. Koza, A. Lewińska, M. Łuczak, P. Malinowski, J. Wołoszyńska, and A. Zielonka for their help in running Studies 1 and 2. We also thank P. Lewandowska, A. Ozimek and members of the Laboratory of Peripheral Psychophysiology at University of Social Sciences and Humanities for their help in data acquisition for Study 3.

Author contributions MW, MP, MR and A. Troszyńska designed all studies, conducted Studies 1 and 2 and analyzed the data from these studies. Aleksandra Tołopiło and MO conducted Study 3 and analyzed the data from this study. MW wrote the original draft. MW, MP and MO edited and revised the draft. All authors reviewed and approved the final manuscript.

Funding The work was supported by the National Science Centre in Poland (Grant Number 2017/26/E/HS6/00725).

\section{Declarations}

Conflict of interest The authors have no conflict of interest to disclose.

Ethical approval The manuscript adheres to the ethical standards of the institutional ethics board as well as ethical principles of the American Psychological Association. The research was approved by the Research Ethics Committee at the University of Lodz, Poland.

Informed consent Informed consent was obtained from each participant. We determined our sample size in advance and report all data exclusions, all manipulations, and all measures. The data that support our findings are publicly available at Open Science Framework.

Open Access This article is licensed under a Creative Commons Attribution 4.0 International License, which permits use, sharing, adaptation, distribution and reproduction in any medium or format, as long as you give appropriate credit to the original author(s) and the source, provide a link to the Creative Commons licence, and indicate if changes were made. The images or other third party material in this article are included in the article's Creative Commons licence, unless indicated otherwise in a credit line to the material. If material is not included in the article's Creative Commons licence and your intended use is not permitted by statutory regulation or exceeds the permitted use, you will need to obtain permission directly from the copyright holder. To view a copy of this licence, visit http://creativecommons.org/licenses/by/4.0/.

\section{References}

Abele, A., \& Wojciszke, B. (2014). Communal and agentic content in social cognition: A dual perspective model. Advances in Experimental Social Psychology, 50, 198-255. https://doi.org/10.1016/ B978-0-12-800284-1.00004-7

Anderson, C., Keltner, D., \& John, O. P. (2003). Emotional convergence between people over time. Journal of Personality and Social Psychology, 84, 1054-1068. https://doi.org/10.1037/00223514.84 .5 .1054

Balsters, M. J. H. (2015). Pro-social effects of ingroup and outgroup tears. Paper presented at the Bi-Annual Conference of the International Society for Research on Emotion, Geneva, Switzerland.
Blechert, J., Peyk, P., Liedlgruber, M., \& Wilhelm, F. H. (2016). ANSLAB: Integrated multichannel peripheral biosignal processing in psychophysiological science. Behavior Research Methods, 48, 1528-1545. https://doi.org/10.3758/s13428-015-0665-1

Bobowik, M., Doroszuk, M., Slawuta, P., \& Basabe, N. (2020). When they cry: Tears facilitate responses toward members of socially disadvantaged groups. Retrieved March 15, 2021, from https:// psyarxiv.com/7gby $3 /$

Bourgeois, P., \& Hess, U. (2008). The impact of social context on mimicry. Biological Psychology, 77, 343-352. https://doi.org/10. 1016/j.biopsycho.2007.11.008

Cicchetti, D. V. (1994). Guidelines, criteria, and rules of thumb for evaluating normed and standardized assessment instruments in psychology. Psychological Assessment, 6, 284-290. https://doi. org/10.1037/1040-3590.6.4.284

Cohn, J. F., \& Ekman, P. (2005). Measuring facial action by manual coding, facial EMG, and automatic facial image analysis. In J. A. Harrigan, R. Rosenthal, \& K. Scherer (Eds.), Series in affective science. The new handbook of methods in nonverbal behavior research (pp. 9-64). Oxford University Press.

Dimberg, U., \& Thunberg, M. (2012). Empathy, emotional contagion, and rapid facial reactions to angry and happy facial expressions. PsyCh Journal, 1, 118-127. https://doi.org/10.1002/pchj.4

Ekman, P., Friesen, W. V., \& Hager, J. C. (2002). Facial action coding system. Research Nexus, Network Research Information.

Elfenbein, H. A. (2014). The many faces of emotional contagion: An affective process theory for affective linkage. Organizational Psychology Review, 4, 326-362. https://doi.org/10.1177/20413 86614542889

Enders, C. K. (2011). Analyzing longitudinal data with missing values. Rehabilitation Psychology, 56, 267-288. https://doi.org/10.1037/ a0025579

Epstude, K., \& Mussweiler, T. (2009). What you feel is how you compare: How comparisons influence the social induction of affect. Emotion, 1, 1-14. https://doi.org/10.1037/a0014148

Faul, F., Erdfelder, E., Lang, A. G., \& Buchner, A. (2007). G*Power 3: A flexible statistical power analysis program for the social, behavioral, and biomedical sciences. Behavior Research Methods, 39, 175-191. https://doi.org/10.3758/bf03193146

Fischer, A. H., \& Hess, U. (2017). Mimicking emotions. Current Opinion in Psychology, 17, 151-155. https://doi.org/10.1016/j.copsyc. 2017.07.008

Fischer, A. H., Kret, M. E., \& Broekens, J. (2018). Gender differences in emotion perception and self-reported emotional intelligence: A test of the emotion sensitivity hypothesis. PLOS ONE, 13, e0190712. https://doi.org/10.1371/journal.pone.0190712

Fischer, A. H., \& Manstead, A. S. R. (2008). Social functions of emotion and emotion regulation. In M. Lewis, J. Haviland, \& L. Feldman Barrett (Eds.), Handbook of emotion (3rd ed., pp. 456-468). Guilford Press.

Fiske, S. T., Cuddy, A. J. C., \& Glick, P. (2007). Universal dimensions of social cognition: Warmth and competence. Trends in Cognitive Sciences, 11, 77-83. https://doi.org/10.1016/j.tics.2006.11.005

Fiske, S. T., Cuddy, A. J. C., Glick, P., \& Xu, J. (2002). A model of (often mixed) stereotype content: Competence and warmth respectively follow from perceived status and competition. Journal of Personality and Social Psychology, 82, 878-902. https://doi.org/ 10.1037//0022-3514.82.6.878

Fridlund, A. J. (1994). Human facial expression: An evolutionary view. Academic.

Friesen, W., \& Ekman, P. (1983). EMFACS-7: Emotional facial action coding system. University of California.

Hareli, S., \& Hess, U. (2012). The social signal value of emotions. Cognition and Emotion, 26, 385-389. https://doi.org/10.1080/ 02699931.2012 .665029 
Hatfield, E., Cacioppo, J. T., \& Rapson, L. R. (1994). Emotional Contagion. Cambridge University Press.

Hess, U. (2021). Who to whom and why: The social nature of emotional mimicry. Psychophysiology, 58, e13675. https://doi.org/10. 1111/psyp. 13675

Hess, U., Arslan, R., Mauersberger, H., Blaison, C., Dufner, M., Denissen, J. J., \& Ziegler, M. (2017). Reliability of surface facial electromyography. Psychophysiology, 54, 12-23. https://doi.org/ 10.1111/psyp. 12676

Hess, U., \& Blairy, S. (2001). Facial mimicry and emotional contagion to dynamic emotional facial expressions and their influence on decoding accuracy. International Journal of Psychophysiology, 40, 129-141. https://doi.org/10.1016/s0167-8760(00)00161-6

Hess, U., Blairy, S., \& Kleck, R. E. (2000). The influence of facial emotion displays, gender, and ethnicity on judgments of dominance and affiliation. Journal of Nonverbal Behavior, 24, 265283. https://doi.org/10.1023/A:1006623213355

Hess, U., \& Fischer, A. (2014). Emotional mimicry: Why and when we mimic emotions. Social and Personality Psychology Compass, 8 , 45-57. https://doi.org/10.1111/spc3.12083

Hess, U., \& Fischer, A. H. (2013). Emotional mimicry as social regulation. Personality and Social Psychology Review, 17, 142-157. https://doi.org/10.1177/1088868312472607

Hsee, C. K., Hatfield, E., Carlson, J. G., \& Chemtob, C. (1990). The effect of power on susceptibility to emotional contagion. Cognition and Emotion, 4, 327-340. https://doi.org/10.1080/02699 939008408081

Izard, C. E., Dougherty, F. E., Bloxom, B. M., \& Kotsch, N. E. (1974). The Differential Emotions Scale: A method of measuring the subjective experience of discrete emotions. Vanderbilt University.

Keltner, D., \& Haidt, J. (1999). Social functions of emotions at four levels of analysis. Cognition and Emotion, 13, 505-521. https:// doi.org/10.1080/026999399379168

Koo, T. K., \& Li, M. Y. (2016). A guideline of selecting and reporting intraclass correlation coefficients for reliability research. Journal of Chiropractic Medicine, 15, 155-163. https://doi.org/10.1016/j. jcm.2016.02.012

Lehane, C. M. (2015). Male and female differences in nonconscious mimicry: A systematic review. Journal of European Psychology Students, 6, 34-48. https://doi.org/10.5334/jeps.de

Likowski, K. U., Mühlberger, A., Seibt, B., Pauli, P., \& Weyers, P. (2008). Modulation of facial mimicry by attitudes. Journal of Experimental Social Psychology, 44, 1065-1072. https://doi.org/ 10.1016/j.jesp.2007.10.007

Mauss, I. B., \& Robinson, M. D. (2009). Measures of emotion: A review. Cognition and Emotion, 23, 209-237. https://doi.org/10. 1080/02699930802204677

Olszanowski, M., Parzuchowski, M., \& Szymków, A. (2019). When the smile is not enough: The interactive role of smiling and facial characteristics in making judgments about perceived trustworthiness and dominance. Annals of Psychology, 22, 35-52. https://doi. org/10.18290/rpsych.2019.22.1-3

Olszanowski, M., Pochwatko, G., Kuklinski, K., Scibor-Rylski, M., Lewinski, P., \& Ohme, R. (2015). Warsaw set of emotional facial expression pictures: A validation study of facial display photographs. Frontiers in Psychology, 5, 1516. https://doi.org/10.3389/ fpsyg.2014.01516

Olszanowski, M., Wróbel, M., \& Hess, U. (2020). Mimicking and sharing emotions: A re-examination of the link between facial mimicry and emotional contagion. Cognition and Emotion, 34, 367-376. https://doi.org/10.1080/02699931.2019.1611543

Parkinson, B. (2005). Do facial movements express emotions or communicate motives? Personality and Social Psychology Review, 9, 278-311. https://doi.org/10.1207/s15327957pspr0904_1
Prochazkova, E., \& Kret, M. E. (2017). Connecting minds and sharing emotions through mimicry: A neurocognitive model of emotional contagion. Neuroscience and Biobehavioral Reviews, 80, 99-114. https://doi.org/10.1016/j.neubiorev.2017.05.013

Ratcliff, N. J., Franklin, R. G., Jr., Nelson, A. J., \& Vescio, T. K. (2012). The scorn of status: A bias toward perceiving anger on high-status faces. Social Cognition, 30, 631-642. https://doi.org/ 10.1521/soco.2012.30.5.631

Rees, L., Friedman, R., Olekalns, M., \& Lachowicz, M. (2020). Limiting fear and anger responses to anger expressions. International Journal of Conflict Management, 31, 581-605. https://doi.org/10. 1108/IJCMA-01-2019-0016

Robinson, M. D., \& Clore, G. L. (2002). Belief and feeling: Evidence for an accessibility model of emotional self-report. Psychological Bulletin, 128, 934-960. https://doi.org/10.1037/0033-2909. 128.6.934

Sachisthal, M. S. M., Sauter, D. A., \& Fischer, A. H. (2016). Mimicry of ingroup and outgroup emotional expressions. Comprehensive Results in Social Psychology, 1, 86-105. https://doi.org/10.1080/ 23743603.2017.1298355

Sato, W., Fujimura, T., Kochiyama, T., \& Suzuki, N. (2013). Relationships among facial mimicry, emotional experience, and emotion recognition. PLoS ONE, 8, e57889. https://doi.org/10.1371/journ al.pone.0057889

Sayette, M. A., Cohn, J. F., Wertz, J. M., Perrott, M. A., \& Parrott, D. J. (2001). A Psychometric evaluation of the facial action coding system for assessing spontaneous expression. Journal of Nonverbal Behavior, 25, 167-185. https://doi.org/10.1023/A:1010671109788

Seibt, B., Mühlberger, A., Likowski, K. U., \& Weyers, P. (2015). Facial mimicry in its social setting. Frontiers in Psychology, 6, 1122. https://doi.org/10.3389/fpsyg.2015.01122

Spoor, J. R., \& Kelly, J. R. (2009). Mood convergence in dyads: Effects of valence and leadership. Social Influence, 4, 282-297. https:// doi.org/10.1080/15534510902805366

Springer, U. S., Rosas, A., McGetrick, J., \& Bowers, D. (2007). Differences in startle reactivity during the perception of angry and fearful faces. Emotion, 7, 516-525. https://doi.org/10.1037/15283542.7.3.516

Tassinary, L. G., Cacioppo, J. T., \& Vanman, E. J. (2007). The skeletomotor system: Surface electromyography. In J. T. Cacioppo, L. G. Tassinary, \& G. G. Berntson (Eds.), Handbook of psychophysiology (pp. 267-299). Cambridge University Press.

Van Boxtel, A. (2010). Facial EMG as a tool for inferring affective states. In A. J. Spink, F. Grieco, O. E. Krips, L. W. S. Loijens, L. P. J. J. Noldus, \& P. H. Zimmerman (Eds.), Proceedings of measuring behavior (pp. 104-108). Eindhoven.

Van der Schalk, J., Fischer, A., Doosje, B., Wigboldus, D., Hawk, S., Rotteveel, M., \& Hess, U. (2011). Convergent and divergent responses to emotional displays of ingroup and outgroup. Emotion, 11, 286-298. https://doi.org/10.1037/a0022582

Van Kleef, G. A., Van Doorn, E. A., Heerdink, M. W., \& Koning, L. F. (2011). Emotion is for influence. European Review of Social Psychology, 22, 114-163. https://doi.org/10.1080/10463283.2011. 627192

Weisbuch, M., \& Ambady, N. (2008). Affective divergence: Automatic responses to others' emotions depend on group membership. Journal of Personality and Social Psychology, 95, 1063-1079. https:// doi.org/10.1037/a0011993

Westfall, J. (2016). PANGEA: Power analysis for general ANOVA designs. Unpublished manuscript. Retrieved November 10, 2019, from http://jakewestfall.org/publications/pangea.pdf

Weyers, P., Mühlberger, A., Kund, A., Hess, U., \& Pauli, P. (2009). Modulation of facial reactions to avatar emotional faces by nonconscious competition priming. Psychophysiology, 46, 328-335. https://doi.org/10.1111/j.1469-8986.2008.00771.x 
Wojciszke, B., Abele, A. E., \& Baryła, W. (2009). Two dimensions of interpersonal attitudes: Liking depends on communion, respect depends on agency. European Journal of Social Psychology, 39, 973-990. https://doi.org/10.1002/ejsp.595

Wojciszke, B., Bazinska, R., \& Jaworski, M. (1998). On the dominance of moral categories in impression formation. Personality and Social Psychology Bulletin, 24, 1245-1257. https://doi.org/ 10.1177/01461672982412001

Wróbel, M., \& Imbir, K. (2019). Broadening the perspective on emotional contagion and emotional mimicry: The correction hypothesis. Perspectives on Psychological Science, 14, 437-451. https:// doi.org/10.1177/1745691618808523

Wróbel, M., \& Królewiak, K. (2017). Do we feel the same way if we think the same way? Shared attitudes and the social induction of affect. Basic and Applied Social Psychology, 39, 19-37. https:// doi.org/10.1080/01973533.2016.1227709

Wróbel, M., \& Olszanowski, M. (2019). Emotional reactions to dynamic morphed facial expressions: A new method to induce emotional contagion. Annals of Psychology, 22, 91-102. https:// doi.org/10.18290/rpsych.2019.22.1-6

Wróbel, M., Rzeczkowska, M., Troszczyńska, A., \& Piórkowska, M. (2020). The "Big Two" and emotional contagion: Interactive effects of agency and communion on socially induced emotions. Manuscript under review.

Zhou, Q., Valiente, C., \& Eisenberg, N. (2003). Empathy and its measurement. In S. J. Lopez \& C. R. Snyder (Eds.), Positive psychological assessment: A handbook of models and measures (pp. 269-284). American Psychological Association. https://doi.org/ $10.1037 / 10612-017$

Publisher's Note Springer Nature remains neutral with regard to jurisdictional claims in published maps and institutional affiliations. 